\title{
Robust and Probabilistic Failure-Aware Placement
}

\author{
MADHUKAR KORUPOLU, Nvidia Inc \\ RAJMOHAN RAJARAMAN, Northeastern University
}

\begin{abstract}
Motivated by the growing complexity and heterogeneity of modern data centers, and the prevalence of commodity component failures, this article studies the failure-aware placement problem of placing tasks of a parallel job on machines in the data center with the goal of increasing availability. We consider two models of failures: adversarial and probabilistic. In the adversarial model, each node has a weight (higher weight implying higher reliability) and the adversary can remove any subset of nodes of total weight at most a given bound $W$ and our goal is to find a placement that incurs the least disruption against such an adversary. In the probabilistic model, each node has a probability of failure and we need to find a placement that maximizes the probability that at least $K$ out of $N$ tasks survive at any time.

For adversarial failures, we first show that (i) the problems are in $\Sigma_{2}$, the second level of the polynomial hierarchy; (ii) a variant of the problem that we call RoBustFAP (for Robust Failure-Aware Placement) is co-NP-hard; and (iii) an all-or-nothing version of RoBustFAP is $\Sigma_{2}$-complete. We then give a polynomialtime approximation scheme (PTAS) for RoBUstFAP, a key ingredient of which is a solution that we design for a fractional version of RoBUstFAP. We then study HierRoBUStFAP, which is the fractional RoBustFAP problem over a hierarchical network, in which failures can occur at any subset of nodes in the hierarchy, and a failure at a node can adversely impact all of its descendants in the hierarchy. To solve HiERRoBustFAP, we introduce a notion of hierarchical max-min fairness and a novel Generalized Spreading algorithm, which is simultaneously optimal for every upper bound $W$ on the total weight of nodes that an adversary can fail. These generalize the classical notion of max-min fairness to work with nodes of differing capacities, differing reliability weights, and hierarchical structures. Using randomized rounding, we extend this to give an algorithm for integral HierRoBUStFAP.

For the probabilistic version, we first give an algorithm that achieves an additive $\varepsilon$ approximation in the failure probability for the single level version, called ProBFAP, while giving up a $(1+\varepsilon)$ multiplicative factor in the number of failures. We then extend the result to the hierarchical version, HIERProBFAP, achieving an $\varepsilon$ additive approximation in failure probability while giving up an $(L+\varepsilon)$ multiplicative factor in the number of failures, where $L$ is the number of levels in the hierarchy.
\end{abstract}

CCS Concepts: • Theory of computation $\rightarrow$ Scheduling algorithms; Stochastic approximation;

Additional Key Words and Phrases: Failure-aware placement, datacenter networks, max-min fairness

Part of this work was done when the first author was at Google Research and the second author was a visiting scientist at Google. This work has been supported by NSF grants CSR-1217981, CCF-1422715, and AITF-1535929, and a Google research award. A preliminary version of this article appeared in Proceedings of the ACM Symposium on Parallelism in Algorithms and Architectures 2016 (Korupolu and Rajaraman 2016).

Authors' addresses: M. Korupolu, Nvidia Inc, Santa Clara, CA; email: madhukar@nvidia.com; R. Rajaraman, College of Computer and Information Science, Northeastern University, Boston, MA; email: rraj@ccs.neu.edu.

Permission to make digital or hard copies of all or part of this work for personal or classroom use is granted without fee provided that copies are not made or distributed for profit or commercial advantage and that copies bear this notice and the full citation on the first page. Copyrights for components of this work owned by others than ACM must be honored. Abstracting with credit is permitted. To copy otherwise, or republish, to post on servers or to redistribute to lists, requires prior specific permission and/or a fee. Request permissions from permissions@acm.org.

(C) 2018 ACM 2329-4949/2018/06-ART5 $\$ 15.00$

https://doi.org/10.1145/3210367

ACM Transactions on Parallel Computing, Vol. 5, No. 1, Article 5. Publication date: June 2018. 
ACM Reference format:

Madhukar Korupolu and Rajmohan Rajaraman. 2018. Robust and Probabilistic Failure-Aware Placement. ACM Trans. Parallel Comput. 5, 1, Article 5 (June 2018), 30 pages.

https://doi.org/10.1145/3210367

\section{INTRODUCTION}

Modern datacenters are becoming increasingly complex and heterogeneous (Barroso and Hölzle 2009; Bodik et al. 2012; Delimitrou and Kozyrakis 2013; Reiss et al. 2012). Machines, racks, network switches, storage drives, and other hardware equipment of different generations and capabilities often co-exist in the data center, leading to heterogeneity both in capacities as well as failure characteristics. The use of commodity hardware and the large scale of these data centers makes failures a common occurence and software systems need to plan and design for them (Barroso and Hölzle 2009; Schroeder and Gibson 2007; Verma et al. 2015).

In fact, the book on warehouse scale computing (Barroso and Hölzle 2009) devotes a whole chapter to failure and fault tolerance. Machines in the data center are often arranged in hierarchies (Al-Fares et al. 2008; Bodik et al. 2012; Sedaghat et al. 2016), with racks and top-of-rack (ToR) switches, which are then inter-connected via network and power hierarchies. The components of these hierarchies are also prone to failure-due to hardware defects, and planned and unplanned outages.

Several recent systems papers address the question of failures and the need to improve reliability in the presence of failures. Ford et al. (2010), Pinheiro et al. (2007), and Schroeder et al. (2009) analyze failures in large disk drives and distributed installations. Gill et al. (2011), Schroeder and Gibson (2007), Vishwanath and Nagappan (2010), and Yalagandula et al. (2004) analyze failures of machines and network elements in large data centers, including Microsoft data centers over the course of a year. Bodik et al. (2012), Sedaghat et al. (2016), and Tang and Iyer (1992) study correlated failures in data centers due to failure of common nodes such as network and power domains. Decreased availability due to failures causes service downtimes and also hurts reputation for cloud service providers.

In recent work, Bodik et al. (2012) and Sedaghat et al. (2016) addressed the problem of placing tasks of a parallel job (such as MapReduce (Dean and Ghemawat 2008), Spark (Zaharia et al. 2010)) among machines in a hierarchical data center so as to increase availability. Weil et al. (2006) addresses placing data chunks in a storage hierarchy using user specified rules and hash functions. However, the treatment is informal and the proposed heuristics are evaluated experimentally without theoretical analysis.

Given this increasingly important context, in this article, we initiate the formal definition of failure-aware placement problem (FAP) and study its computational complexity and approximation algorithms. This models the common scenario in modern data centers where a job (such as MapReduce (Dean and Ghemawat 2008) and Spark (Zaharia et al. 2010)) arrives with multiple identical tasks that need to be placed on machines in the data center. These can be either batch jobs that perform computations over large data sets and terminate when finished or service jobs that run indefinitely serving user requests (Chen et al. 2014; Reiss et al. 2012; Verma et al. 2015). The tasks of the job can be executed in parallel among machines in the data center. Both the machines and the hardware domains that connect them (racks, switches, busducts, power nodes) are prone to failure. Jobs have availability requirements-such as a minimum number of tasks that need to be up and running at any time-to make quick progress and also to meet service level agreements. When a job arrives, the goal of the failure aware placement system is to allocate its tasks among machines so as to improve availability. 
While we present our results in the context of tasks and machines, the concepts are general and apply to storage and other contexts as well. From a storage point of view, failure rates and disk drive characteristics have been studied in Pinheiro et al. (2007), Ford et al. (2010), and Schroeder et al. (2009). Error correcting codes are often used to store data among nodes in such a way that as long as $k$ of $n$ chunks are available, the original data can be reconstructed (Gribaudo et al. 2015; MacWilliams and Sloane 1977; Plank et al. 1997). However, the related problem of how to place the $n$ chunks among the available nodes so as to maximize the probability of $k$ chunks surviving has received little attention (Mills et al. 2015). A good failure-aware placement algorithm can increase data availability and thereby reduce the amount of redundant storage required.

\subsection{Problem Setup}

We formulate two classes of problems for failure-aware placement: robust (adversarial) and probabilistic. The first class of problems focuses on robustness by associating each domain with a weight representing its reliability (higher weight implies higher reliability), and allowing an adversary to remove (i.e., cause failures in) a subset of domains with total weight bounded by a given failure budget $W$. The goal is to determine a placement of $N$ tasks among machines such that the maximum number of tasks that fail, taken over all adversary's choices of failing domains, is minimized. The second class of failure-aware problems considers probabilistic failures by associating a failure probability with each domain. The goal here is to determine a placement of $N$ tasks among machines such that the probability that more than a given number $F$ of tasks fail is minimized.

We study several variants of failure-aware placement under the above two classes. In addition to differences in the nature of failures (adversarial or probabilistic), these variants differ in the organization of the failure domains (single-level or hierarchical), the kind of adversary (fractional or integral), and the kind of placement (fractional or integral). In all of these variants, we focus on the case where all the tasks have the same size. Many of the modern data parallel batch processing systems (such as MapReduce (Dean and Ghemawat 2008) and Spark (Zaharia et al. 2010)) have jobs with multiple (tens to thousands) tasks of the same size running on different machines (Reiss et al. 2012; Verma et al. 2015). They are launched using a config file that specifies the resource requirements for each task and the number of tasks (e.g., see Verma et al. (2015), Section 2.3). Userfacing jobs also often have multiple instances of the same size running on different machines, and they use a load balancer in front to distribute loads evenly among them.

\subsection{Overview of Results and Techniques}

We now formally define the specific problems we study and summarize our results and techniques. For the reader's convenience, we have collected some of the main notation and problem definitions in Table 1.2. In each formulation, we have a set $\mathcal{F}$ of failure domains, numbered 1 through $|\mathcal{F}|$, which include machines and internal nodes in case of a hierarchy, and a job with $N$ identical tasks that just arrived and needs to be placed. Tasks within a job typically have the same size and we infer a capacity $C_{i}$ for each domain, representing the number of tasks of the job that can be placed in the domain. We define a placement as a map $P: \mathcal{F} \rightarrow \mathbb{N}$ with $0 \leq P(i) \leq C_{i}$ for each domain $i$, $0 \leq i \leq|\mathcal{F}|$. For a subset $D$ of disjoint domains, we define $P(D)=\sum_{i \in D} P(i)$.

Adversarial failures. We first consider the robust variants with adversarial failures.

Definition 1.1 (Placement Against Adversarial Failures). Given a non-negative integer $W$, and for each $i$ in $\mathcal{F}$ an integer weight $w_{i}$, the goal of RoBustFAP is to place $N$ tasks subject to capacity constraints so as to minimize the maximum number of tasks placed on any subset of domains with total weight at most $W$. 
Table 1. Notation and Problem Definitions

\begin{tabular}{|c|c|c|}
\hline $\begin{array}{l}\text { Notation or } \\
\text { Problem }\end{array}$ & Short description & Additional remarks \\
\hline$N$ & Number of tasks to be placed & Identical tasks of same job \\
\hline $\mathcal{F}$ & Set of failure domains & Includes machines and internal nodes \\
\hline$C_{i}$ & Capacity of failure domain $i$ & $\begin{array}{l}\text { Number of tasks of the job that can be placed in } \\
\text { domain } i\end{array}$ \\
\hline$P$ & Placement: $\mathcal{F} \rightarrow \mathbb{N}$ & $0 \leq P(i) \leq C_{i}$ for each domain $i, 0 \leq i \leq|\mathcal{F}|$ \\
\hline$P(D)$ & $\begin{array}{l}\text { Number of tasks placed in subset } D \text { of } \\
\text { domains }\end{array}$ & $P(D)=\sum_{i \in D} P(i)$ \\
\hline$w_{i}$ & Reliability weight of failure domain $i$ & Used in RoBustFAP \\
\hline$w(S)$ & Sum of weights of domains in set $S$ & $\left.\sum_{i \in S} w_{(} i\right)$ \\
\hline$W$ & Failure budget in RoBustFAP & Total weight of nodes adversary can remove \\
\hline ROBUSTFAP & $\begin{array}{l}\text { Place } N \text { tasks among } \mathcal{F} \text { such that } \\
\text { maximum number of tasks in any } \\
\text { subset of domains of total weight at } \\
\text { most } W \text { is minimized }\end{array}$ & $\begin{array}{l}\text { Placement } P \text { that optimizes the following } \\
\min _{P} \max _{S: w(S) \leq W} \sum_{i \in S} P(i)\end{array}$ \\
\hline $\begin{array}{l}\text { AllORNONE } \\
\text { RoBUSTFAP }\end{array}$ & $\begin{array}{l}\text { Identical to RoBUstFAP, except that } P \\
\text { is required to be whole: i.e., for every } \\
\text { domain } i, P(i)=0 \text { or } P(i)=C_{i}\end{array}$ & $\begin{array}{l}\text { Whole placement } P \text { that optimizes the following } \\
\min _{P} \max _{S: w(S) \leq W} \sum_{i \in S} P(i)\end{array}$ \\
\hline FracRoBUstFAP & $\begin{array}{l}\text { Identical to RoBustFAP, except that } P \\
\text { can be fractional }\end{array}$ & $\begin{array}{l}\text { Placement } P: \mathcal{F} \rightarrow \mathbb{R} \text { optimizing objective } \\
\min _{P: P(\mathcal{F}) \geq N} \max _{x \in[0,1]^{n}: x \cdot w \leq W} \sum_{1 \leq i \leq n} x_{i} P(i)\end{array}$ \\
\hline$p_{i}$ & Failure probability of domain $i$ & Used in ProbFAP \\
\hline $\operatorname{Pr}_{P}(F)$ & $\begin{array}{l}\text { Probability that number of tasks failing } \\
\text { in } P \text { exceeds } F\end{array}$ & $\sum_{D \subseteq \mathcal{F}: P(D) \geq F} \prod_{i \in D} p_{i} \prod_{i \in \mathcal{F}-D}\left(1-p_{i}\right)$ \\
\hline ProbFAP & $\begin{array}{l}\text { Find placement } P \text { that minimizes } \\
\operatorname{Pr}_{P}(F)\end{array}$ & \\
\hline $\mathcal{T}$ & Failure domain tree & $\begin{array}{l}\text { Machines at leaves and internal nodes } \\
\text { representing other failure domains }\end{array}$ \\
\hline$w_{v}$ & Reliability of node $v$ in $\mathcal{T}$ & \\
\hline$C_{v}$ & Capacity of node $v$ in $\mathcal{T}$ & Number of tasks that can be placed in subtree at $v$ \\
\hline$L$ & Number of levels in $\mathcal{T}$ & Typically 3 to 4 in practice \\
\hline HierRobustFAP & RoBustFAP over hierarchies & \\
\hline HierProbFAP & ProbFAP over hierarchies & \\
\hline
\end{tabular}

For a given set $S$ of domains, let $w(S)$ denote the sum of the weights of the domains in $S$. Formally, the goal of RobustFAP is to find a placement $P$ that optimizes $\min _{P} \max _{S: w(S) \leq W} \sum_{i \in S} P(i)$.

Hardness of RoBustFAP and related problems. As formulated, it is unclear whether RoBUstFAP is in NP because the inner maximization is an NP-complete integer knapsack problem. Since the decision version of RoBUstFAP is of the form $\exists P \forall S$, it is in $\Sigma_{2}$, the second level of the polynomial hierarchy. We establish the following result on the complexity of RoBUSTFAP in Section 2.1.

TheOrem 1.2. The decision version of RoBUStFAP is co-NP-hard.

To better understand the complexity of RoBustFAP, we consider a variant, which we call AllOrNoneRobustFAP, which is identical to RobustFAP, except that we require that the placement $P$ be whole: for any domain $i, P(i)=0$ or $P(i)=C_{i}$; i.e., we either use the entire capacity of a domain or do not use it at all. AlLORNoneRobustFAP belongs to the class of bilevel knapsack problems, which have gathered interest in recent work (Caprara et al. 2013; Dempe and 
Richter 2000; DeNegre 2011). ${ }^{1}$ Building on techniques from Caprara et al. (2013), we obtain the following hardness results for AllOrNoneRoBustFAP in Section 2.2.

Theorem 1.3. AllOrNoneRobustFAP is $\Sigma_{2}$-complete, and NP-hard if the input weights are given in unary form.

Algorithms for RoBUSTFAP and related variants. As a step toward an approximation algorithm for RoBUstFAP, we first consider a fractional variant.

Definition 1.4 (Fractional RobustFAP). The problem FracRobustFAP is identical to RobustFAP, except that the actions of both the placement algorithm and the adversary can be fractional. Formally, we seek a fractional placement $P: \mathcal{F} \rightarrow \mathbb{R}$ optimizing

$$
\min _{P: P(\mathcal{F}) \geq N} \max _{x \in[0,1]^{n}: x \cdot w \leq W} \sum_{1 \leq i \leq n} x_{i} \cdot P(i),
$$

where $n$ is the number of domains in $\mathcal{F}$.

Note that the notion of robustness is different in the RoBUstFAP and FrAcRoBustFAP problems. In RoBustFAP, an adversary can take down any subset of domains with total weight bounded by $W$. In FracRoBustFAP, an adversary can take down any fraction (say $x(i)$ ) of any domain $i$, as a result of which the number of failed tasks in the domain is $x(i)$ times the number of tasks allocated to $i$; the sole constraint is that the total fractional weight of the failed domains, $\sum_{i} x(i) w_{i}$, is bounded by $W$. We show that the classic notion of weighted max-min fair allocation (Keshav 1997; Nagle 1987; Parekh and Gallager 1993) is simultaneously optimal for all weights $W$ for FrAcRoBUSTFAP.

THEOREM 1.5. The weighted max-min fair allocation is simultaneously optimal for all $W$ for FRACROBUSTFAP.

Our main result here is a polynomial-time approximation scheme (PTAS) for RoBUSTFAP that combines the algorithm for FRACROBUSTFAP with a careful enumeration of placements according to how allocations are made to domains. We also present a PTAS for AllOrNoneRobustFAP, which places it alongside a variant of bilevel knapsack called DNEG (Caprara et al. 2013) in the class of $\Sigma_{2}$-complete problems that surprisingly admit polynomial-time approximation schemes.

THEOREm 1.6. There exist polynomial-time approximation schemes for RoBUSTFAP and ALLORNONEROBUSTFAP.

Failure domain hierarchies. Thus far, we have assumed a "flat organization" of the failure domains. We next generalize the problem to failure domain hierarchies, in which we have a failure domain tree $\mathcal{T}$, with machines at the leaves and internal nodes of the tree representing failure domains. Such tree hierarchies are effective models for modern distributed datacenters and have been extensively used in recent studies. Different levels of the hierarchy represent different datacenter elements such as machines, racks, bus ducts, and power nodes (Al-Fares et al. 2008; Bodik et al. 2012; Sedaghat et al. 2016). For storage, failure domains and tree hierarchies with devices, hosts, racks, rows, and so on, are considered in the popular Ceph storage system (cep). We assume that each node $v$ in the hierarchy has a weight $w_{v}$ representing its reliability, and a capacity $C_{v}$ representing the maximum number of tasks that can be placed in the subtree rooted at that node. Failure of an internal node $v$ causes the whole subtree rooted at $v$ and the tasks placed in that

\footnotetext{
${ }^{1}$ A bilevel knapsack problem has two players, each of which controls different aspects of the problem. For example, in one variant, one player sets the capacity of the knapsack, while the other player determines the items to place in the knapsack. In another variant, each player has a knapsack; the first player selects some items for their knapsack and the second player selects items among those not selected by the first player.
} 
subtree to be unavailable. Other topologies, such as fat-trees used in tightly coupled parallel and supercomputers (Leiserson 1985), would be good areas for future study.

Definition 1.7 (Fractional Placement Against Adversarial Failures In Hierarchies). The goal of HierFracRobustFAP is to compute a fractional placement of $N$ tasks among machines in a hierarchy such that the capacity constraints are satisfied and the maximum number of tasks that can fail due to a fractional adversary with weight bound $W$ is minimized.

Hierarchical max-min fairness and Generalized Spreading. While weighted max-min fair allocation works for FRACRoBUSTFAP (Theorem 1.5), the extension to hierarchies needs a hierarchical generalization of weighted max-min fairness, which we provide in Section 4.

Our second main result is a novel Generalized Spreading algorithm, which generalizes the classical notion of uniform spreading among identical nodes to work with nodes of different capacities, different reliability weights, and a hierarchical structure. The Generalized Spreading algorithm yields a hierarchical max-min fair placement that is simultaneously optimal for all weight budget bounds $W$ for HierFracRobustFAP, and could be useful in other domains.

THEOREm 1.8. The Generalized Spreading algorithm is simultaneously optimal for all $W$ for HierFracRobustFAP.

Integral Placements. While the fractional solutions give good intuition and approach for the problem, they end up splitting tasks into fractions and placing them on different nodes, which will not be practical for compute task scenarios. This motivates the need for integral solutions. The rounded-fair placements defined in Section 3 and the corresponding ones for Generalized Spreading in Section 4 are good candidates in practice though they do not have provable computational guarantees like the PTAS algorithms.

For the integral problem HierRoBUSTFAP, we show in Section 4 that a randomized rounding of the Generalized Spreading algorithm solution yields a solution for HiERRoBUSTFAP with performance guarantees against oblivious adversaries, that is, where the failures are determined by an adversary without any knowledge of the placement actions of the algorithm.

Probabilistic failures. The next class of failure-aware placement problems we study is with probabilistic failures: where we model failures by having, for each domain, a probability of failure for that domain.

Definition 1.9 (Placement Under Probabilistic Failures). In ProBFAP, we are given a set of $N$ tasks, and a set of domains $\mathcal{F}$, and for each domain $i$, a probability $p_{i}$ of the domain failing. The goal is to find a placement of tasks to machines subject to capacity constraints so as to minimize the probability that the number of tasks that fail exceeds a given failure bound of $F$.

The probability that the number of tasks failing in a given placement exceeds $F$ is given by

$$
\operatorname{Pr}_{P}(F)=\sum_{D \subseteq \mathcal{F}: P(D) \geq F} \prod_{i \in D} p_{i} \prod_{i \in \mathcal{F}-D}\left(1-p_{i}\right),
$$

where $D$ is the set of domains that fail and the summation is over all possible subsets $D$ of $\mathcal{F}$. The goal of ProbFAP then is to find a placement $P$ that minimizes $\operatorname{Pr}_{P}(F)$.

We then extend the problem formulation to hierarchies of domains. In HierProBFAP, we have a failure domain tree $\mathcal{T}$, each node $v$ of which has a probability $p_{v}$ of failing, and a capacity $C_{v}$ representing the maximum number of tasks that can be placed in the subtree rooted at that node. If a domain $v$ fails, then the entire subtree under it is unavailable, implying that all the tasks placed in the domain fail. Given a bound $F$ on the number of failed tasks, the goal of HierProBFAP is to 
allocate $N$ tasks to the machines in the hierarchy subject to capacity constraints so as to minimize the probability that the number of failed tasks exceeds $F$.

Our main result here is a bicriteria approximation algorithm for ProbFAP and HierProbFAP. Our solution approach builds on the stochastic knapsack framework of Li and Yuan (Li and Yuan 2013) to handle dependencies among knapsack items and hierarchical constraints on the allocations.

Theorem 1.10. There exists a polynomial-time algorithm for HienProbFAP, which computes, for any given constant $\varepsilon>0$, a placement of $N$ tasks such that the probability that the number of failed tasks is at most $(L+\varepsilon) F$ is at least OPT $-\varepsilon$, where OPT is the maximum achievable probability for the given failure bound $F$, and $L$ is the number of levels in the hierarchy.

\subsection{Related Work}

To the best of our knowledge, our specific formulations for failure-aware placement are new. We now review related results in the theory, operations research, and systems communities, and highlight the differences between our work and past work.

Max-min fairness. The notion of max-min fairness and its generalizations, including weighted max-min fairness and generalized processor sharing, have been used in networking (Keshav 1997; Nagle 1987; Parekh and Gallager 1993). The basic idea is to allocate resources among multiple players such that any attempt to further increase the allocation of one player necessarily results in the decrease in allocation of some other player with an equal or smaller allocation. Max-min fairness and its variants are used in process and network schedulers to allow multiple packet flows to share bandwidth fairly (Demers et al. 1989). We show in Section 3.1 that the optimal placement for FrAcRobustFAP is a weighted max-min fair one. For hierarchies, the problem is more involved due to the correlations among the different levels of the hierarchy. To address this, we introduce the concept of hierarchical max-min fairness in the Generalized Spreading algorithm of Section 4.

Bilevel knapsack. The AllOrNoneRobustFAP problem has similarities to the bilevel knapsack problems that model decision makers at multiple levels (Dempe 2002; von Stackelberg 1952). A bilevel knapsack problem has two players: a leader and a follower. First, a leader fixes the values of their variables with the aim of optimizing their objective. Next, the follower reacts by setting their variables with the aim of optimizing their objective, under the constraints imposed by the leader. The variants studied earlier differ in the objective functions and variables under control of the leader and follower (Dempe and Richter 2000; DeNegre 2011; Mansi et al. 2012). Dempe and Richter (Dempe and Richter 2000) study a variant in which the leader controls the capacity of the knapsack while the follower decides which items are packed in the knapsack. In the variant labeled DNeg (DeNegre 2011), both players have a knapsack of their own, and the follower can only choose from items that the leader did not pick. Our AllOrNoneRobustFAP problem has similarity to DNeg (DeNegre 2011); however, the particular combination of constraints and objective functions are different and as a result the complexity results for one do not directly translate to the other. Our $\Sigma_{2}$-completeness and PTAS for AllOrNoneRobustFAP build on ideas from Caprara et al. (2013). The RoBUstFAP problem has a different structure and uses additional ideas.

Stochastic knapsack and chance-constrained optimization. The placement problem under probabilistic failures resembles the category of chance-constrained optimization (Nemirovski and Shapiro 2006; Nikolova 2010), which involves constraints and objectives determined by probability of certain events. One consequence of having such chance constraints is that they define a nonconvex set, making it difficult to apply general optimization techniques (Nemirovski and Shapiro 2006). This challenge applies to ProbFAP and HierProbFAP as well. A number of algorithms have 
been developed for chance-constrained optimization (Agrawal et al. 2012; Bertsimas and Sim 2004; Klopfenstein and Nace 2008; Nikolova 2010; Pagnoncelli et al. 2009); however, these do not yield any provable approximations for our problems of interest. Our solution approach for PROBFAP and HierProbFAP builds on the stochastic optimization framework of Li and Yuan (2013), which uses a Poisson approximation technique (Le Cam 1960). Other recent work on stochastic knapsack and related problems includes Bhalgat et al. (2011) and Li and Deshpande (2011).

Finally, the recent work of Daskalakis et al. (2014) studies a distributed storage problem, which is a stochastic knapsack problem in which the unknown variables that are being determined are real-valued. Using structural results from the study of linear threshold functions, they give a unicriterion additive PTAS for their storage problem. In contrast, we focus on the discrete version of the related placement problem, and obtain a bicriteria additive PTAS for constant-depth network hierarchies with probabilistic failures.

\section{HARDNESS OF PLACEMENT UNDER ADVERSARIAL FAILURES}

In this section, we prove the hardness of RoBustFAP and ALLOrNoneRobustFAP: Theorems 1.2 and 1.3 .

\subsection{Hardness of RoBUStFAP}

Recall that, in the decision version of RoBUSTFAP, our goal is to decide if there exists a placement such that for any subset $S$ of domains with weight at most $W$, the total number of tasks allocated to the domains in $S$ is at most $F$.

Proof of Theorem 1.2: Our co-NP-hardness proof is by a reduction from the NP-hard partition problem (Garey and Johnson 1979). Let $I$ be an instance of the partition problem consisting of a set $S$ of $n$ integers $\left\{a_{i}: 1 \leq i \leq n\right\}$ such that $\sum_{i} a_{i}$ equals an even number $2 W$. The goal of the partition problem is to determine if there exists a subset $T$ of $S$ such that the sum of the elements in $T$ equals $W$.

Given $I$, we construct the following instance $I^{\prime}$ of RoBustFAP. We have one domain for each element of $S$, with the capacity $C_{i}$ and the reliability weight $w(i)$ of the $i$ th domain both equal to $a_{i}$, for $1 \leq i \leq n$. We set (i) $N$, number of tasks we need to place, to $2 W$; (ii) the adversary's weight budget to $W$; and (iii) $F$, the maximum number of allowed failures, to $W-1$.

We now show that $I^{\prime}$ has a placement with maximum failures at most $W-1$ if and only if $I$ cannot be partitioned into two sets of equal size. First, note that there is exactly one feasible placement of $N$ tasks: place $a_{i}$ tasks in domain $i$, as $\sum_{i} a_{i}=N=2 W$. Also, for each subset $X$ of domains, the number of tasks allocated as well as the weight are both equal to $\sum_{i \in X} a_{i}$. If this placement has maximum failures at most $W-1$, then it must be the case all subsets of domains of total weight at most $W$ have weight at most $W-1$; that is, there is no selection of domains of weight $W$. This implies that $I$ cannot be partitioned into two sets of equal size. For the other direction, suppose $I$ cannot be partitioned into two sets of equal size. Since the $\sum_{i} a_{i}=2 W$, it follows that every subset of $S$ that has total size at most $W$ has, in fact, total size at most $W-1$. Thus, in the placement for the instance $I^{\prime}$, any subset of domains of total weight at most $W$ has total weight at most $W-1$, implying that the placement incurs maximum failures of at most $W-1$. We thus obtain that a placement with maximum failures $W-1$ exists if and only if $I$ cannot be partitioned into two sets of equal size, thus establishing the co-NP-hardness.

\subsection{Hardness of AllOrNoneRobustFAP}

We now establish Theorem 1.3, the hardness of AllOrNoneRobustFAP. Clearly, AllOrNoneROBUSTFAP is in $\Sigma_{2}$. We show that it is $\Sigma_{2}$-hard via a reduction from the $\Sigma_{2}$-complete problem 
SubsetSuminterval. Our proof is similar to the proof given in Caprara et al. (2013), which establishes this result for DNEG, a different variant of bilevel knapsack, attributed to DeNegre (2011), which is incomparable to AllOrNoneRobustFAP.

An instance of SubsetSumInterval consists of elements $q_{1}, \ldots, q_{n}$, integers $R$, and $r \leq n$. We ask the question: is there an integer $S$ with $R \leq S<R+2^{r}$ such that no subset $I \subseteq\{1, \ldots, n\}$ satisfies $\sum_{i \in I} q_{i}=S$ ? It was shown in work (Caprara et al. 2013) that SubsetSumInterval is $\sum_{2}$ hard. It is more convenient for us to work with a variant of SubSETSumInTERval, which we call $k$-SubsetSuminterval. An instance of $k$-SubsetSumInterval is an instance of SubSetSumINTERVAL with an additional parameter $k$ and the question asked is: is there an integer $S$ with $R \leq S<R+2^{r}$ such that no subset $I \subseteq\{1, \ldots, n\}$ with $|I|=k$ satisfies $\sum_{i \in I} q_{i}=S$ ?

LEMMA 2.1. $k$-SuBSETSUMINTERVAL is $\Sigma_{2}$-complete.

Proof. The membership of $k$-SubsetSuminterval in $\Sigma_{2}$ is immediate. We establish $\Sigma_{2}$ hardness by a reduction from SubSETSumInTERval. Given an instance of SubSETSumInTERVAL, we construct the following instance of $k$-SubsetSumInterval. We have $2 n$ elements in the $k$ SubsetSumInterval instance: the first $n$ are $q_{i}+1$, for $1 \leq i \leq n$, and the next $n$ are 1 for $0 \leq i<n$; we set $k$ to $n$.

We claim that an integer $S$ satisfies the condition for the SuBSETSUMInTERVAL instance if and only if $S+n$ satisfies the condition for the $k$-SubSETSUmInterval instance. If $S$ is such that $\sum_{i \in I} q_{i}=S$, then consider $I^{\prime}=I \cup\{i: n+1 \leq i \leq 2 n-|I|\}$. We have $\sum_{i \in I^{\prime}} q_{i}^{\prime}=S+n$. Also, note that $\left|I^{\prime}\right|=n$. If $S+n$ is such that $\sum_{i \in I^{\prime}} q_{i}^{\prime}=S+n$ with $\left|I^{\prime}\right|=n$, then letting $I=I^{\prime} \cap\{1, \ldots, n\}$, we have

$$
\sum_{i \in I} q_{i}=\left(\sum_{i \in I} q_{i}^{\prime}\right)-|I|=S+|I|-|I|=S .
$$

We thus have a polynomial time reduction from SubSETSumInTERval to $k$-SubSETSumInterval, establishing the $\Sigma_{2}$-hardness of $k$-SubSETSUmINTERVAL.

Proof of Theorem 1.3: Our proofs for the $\Sigma_{2}$-completeness of AllOrNoneRobustFAP and the NP-hardness for the unary version are similar to that of Caprara et al. (2013) for the bilevel knapsack problem DNEG.

$\Sigma_{2}$-completeness. The membership in $\Sigma_{2}$ follows immediately from the structure of the problem. Suppose we are given an instance $I$ of the $k$-SubsetSumInTERval problem. We introduce three kinds of domains in the AllOrNoneRobustFAP instance.

For each element $q_{i}$ in the $k$-SUBSETSUmInTERval instance, we have an ordinary domain $i$, with $C_{i}=w_{i}=q_{i}+P$, for an integer $P>2^{r}-1+2 r Q$, where $Q=\sum_{1 \leq i \leq n} q_{i}$.

For $1 \leq j \leq r$, we have a padding domain $n+j$ with $C_{n+j}=w_{n+j}=Q+2^{j}$.

For $1 \leq j \leq r$, we have a dummy domain $n+r+j$ with $C_{n+r+j}=w_{n+r+j}=Q$.

We choose $N$ to be $n P+(r+1) Q$, and $W$ to be $k P+R+2^{r}-1+r Q$. We claim that there exists a placement with at least $N$ tasks for which any $W$-bounded adversary can cause less than $W$ failures if and only if the given $k$-SUBSETSUMINTERVAL instance is a YES instance.

We consider two cases. Suppose the $k$-SUBSETSUmInTERvAL instance is a YES instance; so there exists an $S$ such that $S$ is not the sum of any subset of $k$ integers in the instance. Then, consider the placement that selects all the ordinary domains and a subset of the padding and dummy domains whose weights add up to $r Q+R-S+2^{r}-1$. The total capacity of these domains equals $n P+(r+$ 1) $Q+R+2^{r}-1-S$. Since $S \leq R+2^{r}-1$, it follows that the total capacity of the domains selected is at least $N$.

If the adversary does not select all of the padding and dummy domains, then the maximum failures they can cause is less than $k P+Q+(r-1) Q+R-S+2^{r}-1 \leq W$, since the number of 
ordinary domains that the adversary can select is at most $k$. Otherwise, the number of failures caused (and the weight of the domains picked) is $r Q+R-S+2^{r}-1+T$, where $T$ is the total capacity of the ordinary domains that the adversary picks. If the adversary picks fewer than $k$ ordinary domains, then the number of failures falls short of $F$ since the total capacity of all the padding and dummy domains is less than the capacity of a single ordinary domain. Furthermore, due to the weight constraint, the maximum number of ordinary domains that the adversary can pick is $k$. The weight constraint requires that the weight of $T$ be at most $S+k P$. Since no subset of the $k q_{i}$ 's adds up to exactly $S$, it follows that the weight of these domains is strictly less than $k P+S$, implying that the number of failures caused is strictly less than $k P+r Q+R+2^{r}-1=W$, as desired.

Now consider the case where the $k$-SubSETSumInTERVAL instance is a NO instance. Owing to the lower bound on $N$, any placement has to select all the $n$ ordinary domains since the total capacity of all the padding and dummy domains is less than the capacity of a single ordinary domain. We next claim that at least $r$ of the padding and dummy domains have to be selected in the placement; otherwise, the number of tasks placed is less than $n P+Q+(r-1) Q+2^{r}-1 \leq n P+(r+1) Q$. The adversary selects $r$ padding and dummy domains, by picking all of the padding domains in the placement, and the remaining from the dummy domains in the placement. The weight of the domains picked by the adversary and the failures thus caused is $r Q+R+2^{r}-1-S$, for some $R \leq S<R+2^{r}$. Then, the adversary selects a set $X$ of $k$ ordinary domains such that $\sum_{i \in X} q_{i}=S$; such an $X$ exists since we are given a NO instance of $k$-SubsetSumInTERval. The weight of the domains selected equals $k P+r Q+R+2^{r}-1$, equal to the weight bound. The number of failures caused equals $k P+r Q+R+2^{r}-1=W$, as desired. This completes the reduction.

NP-hardness of unary-ALLORNoneRobustFAP. The reduction is from the vertex cover problem, using Sidon sequences. A Sidon sequence is a sequence of integers $s_{1}, s_{2}, \ldots, s_{n}$ such that no two pairs add up to the same number. There exist Sidon sequences such that each element is bounded by $O\left(n^{2}\right)$. Let $S$ equal $\sum_{i} s_{i}$.

Given an instance $G=(V, E)$ of the vertex cover problem, where $|V|=n$, we construct an instance of unary-ALLORNONEROBUSTFAP. We have a domain for each vertex and edge. The capacity and weight of vertex domain $i$ is $n S+s_{i}$. The capacity and weight of edge domain $(i, j)$ is $2 n^{4} S-$ $s_{i}-s_{j}$. If $k$ is the desired size of the vertex cover, then the parameter $N$ is $\left(2 m n^{4}-1\right) S+(n-k) n S$, and $W$ is $2\left(n^{4}+n\right) S$.

If there is a vertex cover of size $k$, then consider the placement that selects all the edge domains and all but the vertex cover domains. The total capacity is at least $2 m n^{4} S-S+(n-k) n S=N$. Any set of selected domains of capacity $W$ needs to include one edge. Once an edge, say $(i, j)$, is selected, the remaining weight is at most $2 n S+s_{i}+s_{j}$. The only two vertices that can give this weight are $i$ and $j$. Since the set of selected domains is the complement of a vertex cover, at least one of $i$ or $j$ is not selected.

For the other direction, suppose there is a placement such that every subset of selected domains of total weight at most $W$ has capacity less than $W$. This implies that there is no subset of selected domains of weight exactly $W$. Since all edge domains have to be selected, this means that there is no edge $(i, j)$ such that both $i$ and $j$ are selected; i.e., for each edge, at least one incident vertex is not selected. The particular value of $N$ implies that at least $n-k$ vertex domains have to be selected. Thus, the complement of this set represents a vertex cover of size at most $k$.

\section{POLYNOMIAL-TIME APPROXIMATION SCHEMES FOR ROBUSTFAP AND ALLORNONEROBUSTFAP}

We first consider the fractional variant FrAcRoBustFAP and show in Section 3.1 that it can be solved optimally using weighted max-min fair allocations. We then present a PTAS for RoBUSTFAP 
Table 2. Notation for RoBustFAP and FracRoBustFAP

\begin{tabular}{|c|c|c|}
\hline $\begin{array}{l}\text { Notation or } \\
\text { definition }\end{array}$ & Short description & Additional remarks \\
\hline Fairness property & $\begin{array}{l}\text { If a domain } i \text { gets less than its "fair } \\
\text { share" compared to another } \\
\text { domain } j \text {, then } i \text { must be full }\end{array}$ & $\begin{array}{l}\text { For any } i \text { and } j, \text { if } \\
P(i) / w_{i}<P(j) / w_{j} \text {, then } P(i)=C_{i}\end{array}$ \\
\hline$N^{\prime}$ & Residual number of tasks & $N^{\prime}=N-\sum_{i} P(i)$ \\
\hline$C_{i}^{\prime}$ & Residual capacity of domain $i$ & $C_{i}^{\prime}=C_{i}-P(i)$ \\
\hline $\begin{array}{l}\text { Full domain } \\
\ln f r(P)\end{array}$ & $\begin{array}{l}\text { Domain } i \text { said to be full for } P \text { if } \\
P(i)=C_{i} \\
\text { Least non-full ratio } P(i) / w_{i} \text { of } P\end{array}$ & $\begin{array}{l}P(i)=C_{i} \text { and } C_{i}^{\prime}=0 \\
\min _{i: P(i)<C_{i}} P(i) / w_{i} . \text { For a fair } \\
\text { placement, all non-full domains } \\
\text { have same ratio. }\end{array}$ \\
\hline $\begin{array}{l}\text { Rounded-fair } \\
\text { placement }\end{array}$ & $\begin{array}{l}\text { Placement obtained by rounding } \\
\text { the fractional min-max fair } \\
\text { placement }\end{array}$ & \\
\hline $\begin{array}{l}\text { Monotonic } \\
\text { placement }\end{array}$ & $\begin{array}{l}\text { For any two domains } i \text { and } j, \\
w_{i}<w_{j} \rightarrow P(i) \leq P(j) \text { or } \\
P(j)=C_{j}\end{array}$ & Weaker condition than fairness \\
\hline Inversion in $P$ & $\begin{array}{l}\text { Pair of domains } i \text { and } j \text { violating } \\
\text { monotonicity property }\end{array}$ & $\begin{array}{l}w_{i}<w_{j}, P(j)<C_{j} \text { and } P(i)>P(j) . \\
\text { For a monotonic placement, } \\
\text { number of inversions is zero }\end{array}$ \\
\hline $\begin{array}{l}\text { Small allocation } \\
\text { for } P\end{array}$ & At most $\lceil 1 / \varepsilon\rceil$ & $P(i) \leq\lceil 1 / \varepsilon\rceil$ \\
\hline $\begin{array}{l}\text { Medium } \\
\text { allocation for } P\end{array}$ & Between $\lceil 1 / \varepsilon\rceil$ and $\varepsilon F$ & $\lceil 1 / \varepsilon\rceil \leq P(i) \leq \varepsilon F$ \\
\hline $\begin{array}{l}\text { Large allocation } \\
\text { for } P\end{array}$ & At least $\varepsilon F$ & $\begin{array}{l}P(i) \geq \varepsilon F \text {, where } F \text { is the target in } \\
\text { the decision version of Robust } F A P\end{array}$ \\
\hline$n$ & Number of domains & $|\mathcal{F}|$ \\
\hline$g(\varepsilon)$ & $\begin{array}{l}\text { Number of values for a large } \\
\text { allocation }\end{array}$ & $\log _{1+\varepsilon}(1 / \varepsilon)=O(\ln (1 / \varepsilon) / \varepsilon)$ \\
\hline$J$ & $\begin{array}{l}\text { Partial monotonic placement with } \\
\text { large allocations filled }\end{array}$ & \\
\hline$K(J)$ & $\begin{array}{l}\text { Set of choices for the adversary } \\
\text { in } J\end{array}$ & $|K(J)| \leq n^{1 / \varepsilon+1}$ \\
\hline$n^{g(\varepsilon)}$ & Number of ways of choosing $J$ & \\
\hline
\end{tabular}

in Section 3.2 using the solution to FracRobustFAP, and a PTAS for AllOrNoneRobustFAP in Section 3.3. For the reader's convenience, we have collected some important notation and definitions used in this section in two tables: Table 3 for RoBustFAP and FracRobustFAP and Table 3.3 for AllOrNoneRobustFAP.

\subsection{FracRobustFAP: Weighted Max-Min Fair Allocations}

We begin with FracRoBustFAP, as defined in Definition 1.4. Consider the weighted max-min fair allocation, a fractional placement that satisfies the following fairness property: if a domain $i$ gets less than its "fair share" compared to another domain $j$, it is only because $i$ is full. More formally, for any $i$ and $j$, if $P(i) / w_{i}<P(j) / w_{j}$, then $P(i)=C_{i}$. Such a placement can be computed by the following 
algorithm, where $N^{\prime}$ denotes the residual number of tasks and $C_{i}^{\prime}$ represents the residual capacity of domain $i$.

1. Initialize residual number of tasks $N^{\prime}=N$ and, for each domain $i$, residual capacity $C_{i}^{\prime}=C_{i}$.

2. Repeat the following until $N^{\prime}=0$ :

1. Let $D=\left\{i: C_{i}^{\prime}>0\right\}$ be the set of non-full domains and $w(D)=\sum_{i \in D} w_{i}$ be their total weight.

2. For each domain $i$ in $D$ : let $a_{i}=\min \left\{C_{i}^{\prime}, N^{\prime} w_{i} / w(D)\right\}$ denote the proportional number of tasks that can be placed on $i$ subject to capacity constraints. Place $a_{i}$ tasks on $i$ and set $C_{i}^{\prime}=C_{i}^{\prime}-a_{i}$

3. Set $N^{\prime}=N^{\prime}-\sum_{i \in D} a_{i}$

Note that the above allocation is a fractional placement. We show that the weighted max-min fair allocation is in fact simultaneously optimal for all weight bounds $W$. This result is somewhat implicit in previous fair allocation works (Nagle 1987; Parekh and Gallager 1993).

For any placement $P$, we define least-non-full-ratio of $P$, denoted $\ln f r(P)$, as the $\min _{i: P(i)<C_{i}}$ $P(i) / w_{i}$, where the minimum is over non-full domains $i$. By definition, in a fair placement $P$, all non-full domains $i$ have the same ratio of $P(i) / w_{i}$. For a placement not satisfying fairness property, there will be a domain $j$ for which $P(j) / w_{j}$ exceeds $\ln f r(P)$.

LEMma 3.1. For any FracRoBustFAP instance with weight bound $W$, there exists an optimal allocation that satisfies max-min fairness.

Proof. Suppose, for the sake of contradiction, that there does not exist any optimal allocation satisfying the fairness property.

Let $P$ be an optimal allocation for the weight bound $W$, with the highest $\ln f r(P)$. Let $i$ denote the arg-min for this ratio minimization, and let $j$ be a domain such that $P(j) / w_{j}$ exceeds $\ln f r(P)$. Since $i$ is non-full, consider an alternative placement $P^{\prime}$ obtained by moving $\varepsilon$ amount from domain $j$ to domain $i$ : in other words, $P^{\prime}(i)=P(i)+\varepsilon$ and $P^{\prime}(j)=P(j)-\varepsilon$, for a sufficiently small $\varepsilon>0$ satisfying $(P(i)+\varepsilon) / w_{i}<(P(j)-\varepsilon) / w_{j}$.

We will now show that $P^{\prime}$ is also an optimal solution with a higher $\ln f r\left(P^{\prime}\right)$, leading to a contradiction. Let $s^{\prime}$ be any knapsack of weight $W$ and value $v^{\prime}$ constructed by adversary for $P^{\prime}$. We'll show that there exists a corresponding knapsack $s$ with the same weight $W$ and value at least $v^{\prime}$ in $P$, thus establishing that $P^{\prime}$ is also optimal.

Consider the knapsack $s$ that is identical to $s^{\prime}$ except that $s_{i}=s_{i}^{\prime}-\varepsilon$ and $s_{j}=s_{j}^{\prime}+\delta w_{i}(P(j)-$ $\varepsilon) /\left(w_{j}(P(i)+\varepsilon)\right)$, where $\delta=\min \left(s_{i}^{\prime}, w_{j}(P(i)+\varepsilon) /\left(w_{i}(P(j)-\varepsilon)\right)\right.$. By construction, $s$ has the same weight as $s^{\prime}$. By our assumption on $\varepsilon, w_{i}(P(j)-\varepsilon) /\left(w_{j}(P(i)+\varepsilon)\right)$ is at least $\delta$, so the value of $s$ is at least that of $s^{\prime}$. Finally, either $\delta=s_{i}^{\prime}$ or $\delta \geq \varepsilon$, and $\delta w_{i}(P(j)-\varepsilon) /\left(w_{j}(P(i)+\varepsilon)\right) \leq \varepsilon$, which together imply that $s$ is a knapsack that can be built from $P$ and has value at least $v^{\prime}$.

Thus, for every knapsack $s^{\prime}$ in $P^{\prime}$, we have a corresponding knapsack in $P$ with at least the same value-implying that $P^{\prime}$ is also optimal and has a higher $\ln f r\left(P^{\prime}\right)$, thus leading to a contradiction and completing the proof.

Lemma 3.2. For any FracRobustFAP instance, there is a unique weighted max-min fair allocation.

Proof. Suppose, for the sake of contradiction, that $P$ and $P^{\prime}$ are two distinct weighted max-min fair allocations. Let $D$ and $D^{\prime}$ be the set of non-full domains in $P$ and $P^{\prime}$ respectively, and let $r$ and $r^{\prime}$ denote the least-non-full-ratios $\ln f r(P)$ and $\ln f r\left(P^{\prime}\right)$, respectively.

If one of $D$ or $D^{\prime}$, say $D$, is empty, then the number $N$ of tasks to allocate exactly equals the sum of the capacities; hence, $D^{\prime}$ also needs to be empty, implying that $P$ and $P^{\prime}$ are, in fact, identical. So, in the remainder, we assume that both are nonempty. Since $P$ and $P^{\prime}$ are fair, all domains $i$ 
in $D$ have $P(i) / w_{i}$ equal to $r$ and all domains $i^{\prime}$ in $D^{\prime}$ have $P^{\prime}\left(i^{\prime}\right) / w_{i}^{\prime}$ equal to $r^{\prime}$. Without loss of generality, assume $r \geq r^{\prime}$.

We argue that $D$ is a subset of $D^{\prime}$; otherwise, there exists an $i$ that is not full in $P$ but full in $P^{\prime}$ and we have $P(i) / w_{i}=r \geq r^{\prime} \geq P^{\prime}(i) / w_{i}=C_{i} / w_{i}$, a contradiction. Since $D$ is a subset of $D^{\prime}, r \geq r^{\prime}$, and the domains not in $D$ are full, every domain has at least as much allocated in $P$ as in $P^{\prime}$. Since $P$ and $P^{\prime}$ are distinct, at least one domain must have more allocated, which is a contradiction since the total size of each allocation is $N$. This completes the proof of the lemma.

Lemmas 3.1 and 3.2 establish the following Theorem.

THEOREM 3.3. For a single level of failure domains with weight vector $\left(w_{1}, \ldots, w_{m}\right)$ and capacity vector $\left(C_{1}, \ldots, C_{m}\right)$, the weighted max-min fair allocation is simultaneously optimal for all weight bounds $W$ for FracRoBustFAP.

\subsection{RobustFAP}

For RoBustFAP, one can easily construct instances to show that no (integral) placement can be simultaneously optimal for all weight bounds. For instance, suppose we need to place $N=2$ tasks among two domains $A$ and $B$ with weight 1 and 2, respectively. The only optimal placement for the weight bound $W=1$ is to assign all tasks to $B$, while the only optimal placement for $W=2$ is to split the tasks between $A$ and $B$.

Given the "universal" optimality of the weighted max-min fair allocation for FrAcRoBustFAP, it is natural to explore its integer variants for RoBUSTFAP. One integer variant of the weighted max-min fair allocation that we consider is the rounded fair placement, defined below.

Definition 3.4. For a given RoBUstFAP instance, we obtain a rounded fair placement by running the following procedure: (i) compute the weighted max-min fair allocation $P_{f}$ for the RoBUSTFAP instance; (ii) let $D$ be the set of domains that have a non-integer assignment, and $k<|D|$ be the integer $\sum_{i \in D}\left(P_{f}(i)-\left\lfloor P_{f}(i)\right\rfloor\right)$; (iii) set the rounded fair placement $P_{f}^{\prime}$ as follows: $P_{f}^{\prime}(i)=\left\lceil P_{f}(i)\right\rceil$ for $i$ belonging to an arbitrary $k$-size subset of $D$, and $P_{f}^{\prime}(i)=\left\lfloor P_{f}(i)\right\rfloor$ for all other $i$.

We immediately observe that $P_{f}^{\prime}(i)<P_{f}(i)+1$ for all $i$. Though the rounded fair placement does not directly yield a PTAS, it plays a central role in our PTAS construction below.

Proof of first half of Theorem 1.6. We now describe our PTAS for RoBustFAP. Recall that the goal of RoBustFAP is to determine a placement $P$ that minimizes the maximum of $P(T)$, over all subsets $T$ of $\mathcal{F}$ with total weight at most $W$, such that (i) $P(\mathcal{F})=N$, and (ii) for each $i \in \mathcal{F}$, $P(i) \leq C_{i}$. Consider the decision version of RoBustFAP: does there exist a placement such that for any subset $T$ of domains whose weight is at most $W$, the sum of tasks assigned to domains in $T$ is at most $F$ ? We give a poly-time algorithm that either determines that there does not exist any such placement, or returns a placement such that for any subset $T$ of $\mathcal{F}$ whose weight is at most $W$, the sum of tasks placed in $T$ is at most $(1+\varepsilon) F$. We then obtain a PTAS for RoBustFAP via a binary search over $F$, invoking the above decision algorithm at each step.

Our decision algorithm proceeds as follows. We first show that it is sufficient to concentrate on a special class of placements, which we refer to as monotonic placements. The number of different monotonic placements is prohibitively large, so we next constrain the placements so that the number of tasks allocated is close to an integer power of $(1+\varepsilon)$. We classify allocations into three categories-large, medium, and small-based on the number of tasks allocated. We then show that a judicious enumeration of large, small, and medium allocations enables computing a near-optimal placement in polynomial time. 
Definition 3.5. We say that a placement $P$ is monotonic if for any two domains $i$ and $j$, if $w_{i}<w_{j}$ and $P(j)<C_{j}$, then $P(i) \leq P(j)$. Note that monotonicity is a weaker requirement than fairness.

Lemma 3.6. There exists a monotonic placement that is optimal for RoBustFAP.

Proof. For any placement $P$, we define the number of inversions of $P$ as the number of pairs $(i, j)$ such that $w_{i}<w_{j}, P(j)<C_{j}$, and $P(i)>P(j)$. The number of inversions lies between 0 and $n(n-1) / 2$, where $n$ is the number of domains. A monotonic placement has no inversions. Let $P$ be an optimal placement that has the least number of inversions. If this number is 0 , we are done. Otherwise, we will derive a new optimal placement that has fewer inversions, thus contradicting the assumption and establishing the lemma.

Let $(i, j)$ be any inversion in $P$. We define a new placement $P^{\prime}$, which is identical to $P$ except that $P^{\prime}(j)=\min \left\{C_{j}, P(i)\right\}$, and $P^{\prime}(i)=P(i)-\left(P^{\prime}(j)-P(j)\right)$. By construction, all capacity constraints are maintained. It is not hard to see that the number of inversions of $P^{\prime}$ is less than that of $P$. We now show that $P^{\prime}$ is also optimal. Let $T^{\prime}$ denote the set of domains selected by the adversary against $P^{\prime}$. Clearly, $w_{T^{\prime}} \leq W$. If $T^{\prime}$ includes both $i$ and $j$, then the adversary can select exactly the same set $T^{\prime}$ against $P$ and achieve the same value. Similarly, if $T^{\prime}$ includes neither $i$ nor $j$, then the adversary can select exactly the same set $T^{\prime}$ against $P$ and achieve the same value. If $T^{\prime}$ includes $i$ but does not include $j$, then the adversary can select exactly the same set $T^{\prime}$ against $P$ and achieve a value of $F^{\prime}-P^{\prime}(i)+P(i)>F^{\prime}$. Finally, if $T^{\prime}$ includes $j$ but does not include $i$, then the adversary can select the set $T^{\prime}-\{j\} \cup\{i\}$, with weight at most $W+w_{i}-w_{j} \leq W$, against $P$ and achieve a value of $F^{\prime}-P^{\prime}(j)+P(i) \geq F^{\prime}$. Thus, the value achieved by the adversary against $P$ is at least that against $P^{\prime}$, thus completing the proof.

Grouping of capacities and allocations. We constrain the placements to only allocate for each domain $i$ a number of tasks that is either at most $\lceil 1 / \varepsilon\rceil$, or the floor of an integer power of $(1+\varepsilon)$, or $C_{i}$.

Definition 3.7. We say that an allocation to a particular domain is small if it is at most $\lceil 1 / \varepsilon\rceil$, large if it is not small and at least $\varepsilon F$, and medium otherwise.

Controlling large allocations. Since a large allocation is at least $\varepsilon F$ and at most $F$, it follows from our grouping of allocations that there are at most $g(\varepsilon)=\log _{1+\varepsilon}(1 / \varepsilon)=O(\ln (1 / \varepsilon) / \varepsilon)$ values to choose from for a large allocation. Since we are restricting to monotonic placements, choosing a large allocation corresponds to choosing $g(\varepsilon)$ stepping points-so there are at most $n^{g(\varepsilon)}$ different ways in which large allocations can be assigned to the domains, where $n$ is the number of domains. Each way yields a partial placement: it specifies the exact allocation for all domains that have large allocations, while the small and medium allocations are undetermined. Given one such partial monotonic solution $J$, the adversary selects a subset of at most $\lceil 1 / \varepsilon\rceil$ domains with large allocations (since each one has at least $\varepsilon F$ ), subject to the weight constraint; let $K(J)$ denote the set of possible choices for the adversary. Thus, for a given $J, K(J)$ has size at most $n^{1 / \varepsilon+1}$. We will use this upper bound on the size of $K(J)$ at the end of the proof when we combine the enumerations over large, small, and medium allocations.

Enumerating small allocations. Restricting to monotonic placements allows us to bound the number of ways in which we can make small allocations. Suppose we sort the domains in nondecreasing order of weights: i.e., $w_{1} \leq \cdots \leq w_{n}$. Then, the small allocations are completely characterized by $t=\lceil 1 / \varepsilon\rceil+1$ stepping indices $0=i_{0} \leq i_{1} \leq \cdots \leq i_{t-1} \leq n$ such that every domain in the interval $\left(i_{j-1}, i_{j}\right)$ has exactly $\min \left\{C_{i}, j\right\}$ tasks. Clearly, the number of ways we can determine these small allocations is at most $n^{t}=n^{\lceil 1 / \varepsilon\rceil+1}$, which is $n^{O(1 / \varepsilon)}$. In the remainder, we fix one choice $S$ of the small allocations. 
Table 3. Notation for AlLOrNoneRobustFAP

\begin{tabular}{|l|l|l|}
\hline \hline Notation & \multicolumn{1}{|c|}{ Short description } & \multicolumn{1}{c|}{ Additional remarks } \\
\hline$S$ & $\begin{array}{l}\text { Subset of } \mathcal{F} \text { on which to place } \\
\text { tasks on wholly }\end{array}$ & For $i \in S, P(i)=C_{i}$ and 0 otherwise \\
\hline$T$ & Subset of $S$ chosen by adversary & $\begin{array}{l}w(T) \leq W \text { and maximize } P(T)= \\
\sum_{i \in T} C_{i}\end{array}$ \\
\hline$X$ & Set of small domains & $\left\{i: C_{i}<\varepsilon F\right\}$ \\
\hline$Y$ & Set of large domains & $\left\{i: C_{i} \geq \varepsilon F\right\}$ \\
\hline$M$ & Total sum of capacities & $C(X)+C(Y)$ \\
\hline$J$ & $\begin{array}{l}\text { Partial solution, subset of } S, \text { chosen } \\
\text { so far }\end{array}$ & $\begin{array}{l}\text { Set of domains dropped from } X \\
\text { based on } J\end{array}$ \\
\hline$K(J)$ & Adversary's selection from $K(J)$ & $\begin{array}{l}\text { Chosen in increasing order of den- } \\
\text { sity } C_{i} / w_{i} .\end{array}$ \\
\hline$E(J)$ & $\begin{array}{l}\text { Adversary's selection from } \\
X-E(J)\end{array}$ & $\begin{array}{l}\text { Max capacity over all } I \in K(J), \text { of } \\
I \cup L(I, J)\end{array}$ \\
\hline$I$ & Adversary's value for $J$ &
\end{tabular}

Determining medium allocations. Thus far, we have fixed a partial placement that assigns large allocations to some domains, and small allocations to others, and the adversary has selected a subset of the domains with large allocations. It remains to assign medium allocations so that the maximum value that can be achieved by the adversary is bounded by $(1+\varepsilon) F$. Let $N^{\prime}$ denote the residual number of tasks to be assigned, and let $\mathcal{F}^{\prime}$ denote the set of domains for which an allocation has not yet been determined. Note that the weight of each domain in $\mathcal{F}^{\prime}$ is at most that of each domain that has already been assigned a large allocation.

We need to consider the value achieved by an adversary that selects domains with medium and small allocations. Since all these domains have at most $\varepsilon F$ tasks, an adversary can achieve a value within an $\varepsilon F$ additive amount of the optimal by ordering the domains in increasing order of their density, and selecting a prefix of this sequence until no more domains can be added. We refer to this adversary as the prefix adversary.

Since we are restricting to medium allocations, we first place $\lceil 1 / \varepsilon\rceil$ tasks in each domain in $\mathcal{F}^{\prime}$, and set the capacity of each domain $i$ in $\mathcal{F}^{\prime}$ to be $\min \left\{\varepsilon F, C_{i}\right\}$. If the number of tasks already placed exceeds $N^{\prime}$, then the current partial assignment is not part of an $(1+\varepsilon)$-optimal solution. Otherwise, we assign the remaining tasks using the rounded fair placement, which assigns to each domain in $\mathcal{F}^{\prime}$ at most one more task than the weighted max-min fair allocation. Since the number of tasks in any medium allocation is at least $\lceil 1 / \varepsilon\rceil$, this additional task per domain with a medium allocation can cause only an extra $\varepsilon$ fraction task failure, as compared with the weighted max-min fair allocation, which is optimal against all fractional adversaries. As we argued above, the prefix adversary is within an additive $\varepsilon F$ of an optimal fractional adversary. Consequently, for medium allocations, the value achieved by the rounded fair placement is within an additive $\varepsilon F$ of optimal against any adversary. 
Putting it together. We enumerate over partial monotonic placements $J$ in which the large allocations are fixed, an adversarial choice from the set $K(J)$ that determines which domains with large allocations to fail, a small allocation $S$, and run the rounded fair placement procedure for medium allocations. For each of these choices, we calculate the number of tasks failed by a weight $W$-bounded adversary, and determine if there exists a solution with at most $(1+\varepsilon) F$ failures. Given the polynomial size of $J, K(J)$, and the number of small allocations, we obtain a PTAS for RoBUsTFAP, thus completing the proof of the first half of Theorem 1.6.

\subsection{AllOrNoneRobustFaP}

Recall that in AllOrNoneRobustFAP, we have a set $\mathcal{F}$ of failure domains, a weight $w_{i}$ and capacity $C_{i}$ for each domain $i$, and an integer $N$. In ALLORNoneRobustFAP, the placements are required to be whole, i.e., $P(i)=0$ or $P(i)=C_{i}$. So the goal of AllOrNoneRobustFAP can be stated as selecting a subset $S$ of $\mathcal{F}$ to place the tasks on such that (a) $\sum_{i \in S} C_{i} \geq N$, and (b) maximum over any subset $T$ of $S$ whose weight is at most $W$, of $\sum_{i \in T} C_{i}$ is minimized.

Proof of second half of Theorem 1.6. As in Section 3.2, we give a polynomial-time approximate algorithm for the decision problem and then give a PTAS for the optimization problem by doing a binary search. The decision problem is the following: given $\mathcal{F}$, and integers $W$ and $F$, is there a subset $S$ of $\mathcal{F}$ such that (a) the capacity if $S$ is at least $N$ and (b) for any subset $T$ of $S$ whose weight is at most $W$, the total capacity of the domains in $T$ is at most $F$ ?

Classifying domains by weight and capacity. We group the domains into multiple categories. The first is where $w_{i}>W$. It is easy to see that these domains can always be included in $S$. So we include these, reduce $N$ as necessary, and continue. The next group of domains is where $C_{i}>F$. It is clear that these domains cannot be included in $S$; otherwise, any such domain can be included in $T$ leading to a total capacity of $T$ to exceed $F$. So, in the remaining, we restrict attention to those domains for which $w_{i} \leq W$ and $C_{i} \leq F$.

Call a domain large if $C_{i} \geq \varepsilon F$, and small, otherwise. Let $X$ denote the set of small domains and $Y$ the set of large domains. We group the large domains into subgroups by rounding each capacity down to the nearest power of $(1+\varepsilon)$. The number of subgroups is $O\left(\log _{1+\varepsilon}(1 / \varepsilon)\right)=$ $O((1 / \varepsilon) \ln (1 / \varepsilon)$.

Controlling the large domains selected by the adversary. We cannot allow the adversary to select more than $1 / \varepsilon$ of the domains from $Y$, all of which are large, since then, the total capacity of the domains selected by the adversary exceeds $F$. We enumerate over every possible subset of $\lfloor 1 / \varepsilon\rfloor$ domains from $Y$, whose total capacity is at most $F$; again, there are at most $n^{1 / \varepsilon}$ such subsets to consider.

Any such set is a possible set of domains selected by the adversary. The adversary will select a particular subset $I$ only if we eliminate, within each subgroup of $Y$, all domains lighter than the lightest domain in $I$ belonging to the same subgroup. Thus, it is sufficient to consider our strategies that eliminate, within each subgroup the $i$ lightest domains, for some $i$. There are at most $n$ choices for this $i$, and at $\operatorname{most} \log _{1+\varepsilon}(1 / \varepsilon)$ subgroups. So in all, we consider $O\left(n^{(1 / \varepsilon) \ln (1 / \varepsilon)}\right)$ solutions. Let $J$ be one such solution. If the algorithm includes $J$ as a subset of $S$, and eliminates all other domains in $Y$ from $S$, then the adversary can select at most $\lceil 1 / \varepsilon\rceil$ lightest remaining domains in each subgroup; hence, there is a constant number of choices for the adversary. More than these many domains will exceed the capacity threshold $F$, and hence, are not needed for the adversary. Since the number of subgroups is $O((1 / \varepsilon) \ln (1 / \varepsilon)$, the number of different choices to consider for the adversary is $(\lceil 1 / \varepsilon\rceil)^{O((1 / \varepsilon) \ln (1 / \varepsilon))}$. Let this set of choices be $K(J)$.

Controlling the small domains selected by the adversary. For any $J$, let $C(J)$ denote the total capacity of the domains in $J$. So, if we eliminated $Y-J$ from $Y$, we can eliminate at most 
$M-N-C(Y-J)$ from the domains in $X$. Within $X$, the adversary is solving a knapsack problem. Since each domain is small, the adversary can select domains in decreasing order of the ratio $C_{i} / w(i)$ and be at most an additive $O(\varepsilon F)$ amount from the optimal. Therefore, we sort the domains in decreasing order of this ratio and eliminate the maximal set of domains of total capacity at most $M-N-C(Y-J)$, and add the remaining to $S$. The capacity of $S$ is thus at least

$$
C(J)+C(X)-M+N+C(Y-J)=C(X)+C(Y)-M+N=N,
$$

as desired. Let this set of eliminated domains be $E(J)$. Since each domain is of capacity at most $\varepsilon F$, we will eliminate at least $M-N-C(J)-\varepsilon F$. Consequently, subject to having selected $I$, the adversary's selection from $X$ would have a capacity of at most $O(\varepsilon F)$ more than an optimal adversary's.

Putting it together. For each $J$, we thus have the following solution $S=J \cup(X-E(J))$. The adversary has $(1 / \varepsilon)^{O\left(\log _{1+\varepsilon}(1 / \varepsilon)\right)}$ choices for $I$ in $K(J)$. Given $J, E(J)$, and $I$, the adversary selects domains from $X \backslash E(J)$ in non-increasing order of the ratio of size to weight, as long as total selected weight is at most $W-w(I)$; let this set of selections be given by $L(I, J)$. Thus, for each $J$, we have $(1 / \varepsilon)^{O\left(\log _{1+\varepsilon}(1 / \varepsilon)\right)}$ choices of the form $I \cup L(I, J)$ for the adversary. Let $v(J)$ denote the maximum capacity, over all $I$, of $I \cup L(I, J)$.

We enumerate over all $J$ and calculate $v(J)$ for each $J$. If there exists a $J$ such that $v(J) \leq F$, then there exists an allocation-specifically, $J \cup E(J)$-such that the adversary can cause at most $(1+O(\varepsilon)) F$ tasks to fail. Otherwise, it follows that for all allocations, there exists an action of the adversary that will cause more than $F$ tasks to fail. Since the number of choices for $J$ is polynomial in the size of the input, and the number of choices of the form $I \cup L(I, J)$, an iteration over which yields $v(J)$, is also polynomial in the size of the input, the total number of enumeration steps is polynomial in the size of the input, yielding a PTAS for AlLORNoneRoBustFAP, thus completing the proof of the second half of Theorem 1.6.

\section{HIERARCHICAL ROBUSTFAP}

We now present the Generalized Spreading algorithm for HierFracRobustFAP in Sections 4.1 and 4.2. We then use the Generalized Spreading algorithm to develop a randomized algorithm for HierRobustFAP in Section 4.3. For the reader's convenience, we have collected some important notation and definitions used in this section in Table 4.1.

\subsection{HierFracRobustFAP: Hierarchical Max-Min Fairness}

Recall that in HierFracRobustFAP, we have a failure domain tree, with machines at the leaves and internal nodes representing failure domains. We now develop a hierarchical notion of maxmin fairness. Given a fractional placement $P$ and node $j$, let $L(j)$ denote the set of all leaves in the subtree rooted at $j$, and $P(j)$ denote $P(L(j))$-i.e., the number of tasks placed by $P$ in the subtree rooted at $j$. We now introduce the notion of vulnerability, an important concept for our algorithm.

Definition 4.1. Given a placement $P$, for any leaf $l$, and any subtree $T$ containing $l$, we define $p_{l}(T, P)$ as follows.

$$
p_{l}(T, P)=\max _{\text {ancestor } j \text { of } l \text { in } T} P(j) / w_{j}
$$

We refer to $p_{l}(T, P)$ as the vulnerability of $l$ in $T$.

Note that this definition generalizes the analogous notion of least-non-full-ratio $(\ln f r)$ used for defining fairness in the single-level case in Section 3.1. To present the generalized notion of fairness, we first define the notion of a full node; informally, a node is full if no more tasks can be assigned to any leaf in the subtree of the node, without violating some capacity constraint. 
Table 4. Notation for Generalized Spreading

\begin{tabular}{|c|c|c|}
\hline $\begin{array}{l}\text { Notation or } \\
\text { definition }\end{array}$ & Short description & Additional remarks \\
\hline$l$ & Leaf node in hierarchy tree & Machines where tasks are placed \\
\hline$j$ & Node in the hierarchy tree & Leaf or internal node \\
\hline$T_{j}$ & Subtree, rooted at $j$, of hierarchy tree & \\
\hline$L(j)$ & Leaf node descendants of $j$ & Leaves in $T_{j}$ \\
\hline$P(j)$ & $\begin{array}{l}\text { Number of tasks placed in the subtree } \\
\text { rooted at } j\end{array}$ & $P(L(j))$ \\
\hline$p_{l}(T, P)$ & $\begin{array}{l}\text { Vulnerability of } l \text { in } T \text { (subtree } \\
\text { containing } l \text { ) with } P\end{array}$ & $\max _{\text {ancestor } j \text { of } l \text { in } T} P(j) / w_{j}$ \\
\hline $\begin{array}{l}\text { Hierarchical } \\
\text { max-min fair }\end{array}$ & $\begin{array}{l}\text { For leaf nodes } l \text { and } l^{\prime} \text {, and any } \\
\text { subtree } T \text { containing } l \text { and } l^{\prime}, \\
p_{l}(T, P)<p_{l}^{\prime}(T, P) \text { implies that } l \text { has a } \\
\text { full ancestor in } T\end{array}$ & Generalizes max-min fair \\
\hline alloc_rates ${ }_{j}$ & $\begin{array}{l}\text { Vector of normalized rates for leaves } \\
\text { in } L(j) \text {, by which we can increment } \\
\text { their allocation (subject to capacity } \\
\text { constraints) }\end{array}$ & $\begin{array}{l}\text { For leaf } l \text {, alloc_rates }{ }_{l} \text { is a scalar set } \\
\text { to } 1\end{array}$ \\
\hline alloc_bound $_{j}$ & $\begin{array}{l}\text { A scalar positive upper bound } \\
\text { indicating the (fractional) number of } \\
\text { tasks that we can allocate using the } \\
\text { relative rates alloc_rates }\end{array}$ & $\begin{array}{l}\text { For leaf } l \text {, alloc_bound } d_{l} \text { is } \\
\text { remaining capacity of } l\end{array}$ \\
\hline $\max \_v u l_{j}(x)$ & $\begin{array}{l}\text { Linear function over scalar variable } x \\
\text { yielding the maximum vulnerability } \\
\text { in the subtree rooted at } j \text { if } x \text { units of } \\
\text { tasks are added to } j\end{array}$ & $\begin{array}{l}\text { For leaf } l, \\
\max \_v u l_{l}(x)=(P(l)+x) / w_{l} .\end{array}$ \\
\hline$H_{j}$ & Non-full children of node $j$ & \\
\hline$r_{j}(x)$ & Ratio $(P(j)+x) / w_{j}$ & \\
\hline
\end{tabular}

Definition 4.2. We say that a leaf node is full if the number of tasks placed on the node equals its capacity. We say that an internal node $j$ is full if either all of its children are full or the total number of tasks placed in the subtree rooted at $j$ equals the capacity of $j$. We say that a node is non-full if it is not full.

Definition 4.3. We say that a placement $P$ is hierarchical max-min fair if for any pair of leaf nodes $l$ and $l^{\prime}$, and any subtree $T$ containing $l$ and $l^{\prime}$, if $p_{l}(T, P)<p_{l^{\prime}}(T, P)$, then there exists a full ancestor of $l$ in $T$. By definition, any hierarchical max-min fair placement, when restricted to any subtree, is also hierarchical max-min fair. For the sake of brevity, we use "fair" (resp., "fairness") and "hierarchical max-min fair" (resp., "hierarchical max-min fairness") synonymously in this section.

In the following lemmas, we show that a hierarchical max-min fair optimal solution exists for every weight bound, and establish the existence of a unique hierarchical max-min fair placement for a given number of tasks; this solution is hence simultaneously optimal for all weight bounds. We defer the proofs of the lemmas to Section 4.4 after we present a polynomial-time algorithm to compute the hierarchical max-min fair placement in Section 4.2.

LEMMA 4.4. For any instance of HIERFRACRoBUstFAP and weight bound $W$, there exists a hierarchical max-min fair optimal solution. 
LEMmA 4.5. For any instance of HIERFRACRoBustFAP, there exists a unique hierarchical max-min fair allocation.

\subsection{Generalized Spreading for HierFracRobustFAP}

We now present an algorithm for computing the hierarchical max-min fair allocation for HIERFracRoBustFAP. Our algorithm incrementally allocates tasks to the leaf nodes, while always maintaining the fairness property. The intuition is best captured by thinking of directing fluid (tasks) into each of the leaf nodes in such a way that the next infinitesimal unit goes to that node which has the least vulnerability. Since vulnerability increases with the assignment of tasks, that node will soon have more vulnerability than some of the other nodes, in which case, the next infinitesimally small unit goes to another node. Thus, in a sense, we ensure that all leaves that are receiving tasks all have the same vulnerability. Repeating this process will yield the desired allocation. How do we convert the above intuition into a finite efficient procedure? The key challenge in implementing this algorithm is that the rate of increase for different leaf nodes is different, depending on the reliability of the ancestor that determines their vulnerability. Note that this rate of increase is not even constant for any given node.

We now present our Generalized Spreading algorithm. The algorithm proceeds by repeating an iteration of steps until all tasks have been allocated. The capacity constraints demand that any leaf node that has a full ancestor cannot receive any more tasks. Following the above intuition, we also ensure that all the leaves in any subtree that are receiving tasks in this spreading procedure will have the same vulnerability; indeed, this will be the maximum vulnerability within the subtree since any leaf that has a lower vulnerability must have an ancestor that is full.

To establish the fairness of our algorithm, we ensure that the algorithm maintains the following invariant:

(I1) The placement at the start of each iteration is hierarchically max-min fair.

Initialization of Generalized Spreading. The initial placement is the empty placement in which the number of tasks assigned to each leaf is 0 . Note that the vulnerability of each leaf in any subtree is 0 . So, by Definition 4.3, the empty placement is fair.

An iteration of Generalized Spreading. Let $P$ be a given current allocation, and we would like to add more tasks. We proceed in a bottom-up manner and compute the following for each non-full node $j$. Let $T_{j}$ denote the subtree rooted at $j$.

(1) Vector alloc_rates $j$ giving the (normalized) rates for the leaves in $T_{j}$ by which we can increment their allocation, subject to capacity constraints; the rates are normalized in the sense that the sum of the coordinates of the vector alloc_rates ${ }_{j}$ is 1 . Each coordinate of the vector represents a rate for a particular leaf in the subtree rooted at $j$. Note that the coordinate for any leaf that has a full ancestor in $T_{j}$ will be 0 .

(2) A scalar upper bound alloc_bound ${ }_{j}>0$ indicating the (fractional) number of tasks that we can allocate using the normalized rates alloc_rates ${ }_{j}$, and

(3) $\max _{-} v u l_{j}(x)$, a linear function over a scalar variable $x$ yielding the maximum vulnerability in the subtree rooted at $j$ if $x$ units of tasks are allocated to the subtree rooted at $j$.

We show how to compute the above three quantities for a non-full node $j$, given the same for non-full children of $j$. To establish invariant (I1), we establish the following:

(I2) At the start of each iteration, for any non-full node $j$ and any non-full children $i$ and $k$ of $j$, $\max _{-} v u l_{i}(0)=\max _{-} v u l_{k}(0)$.

Computation at the leaves. For any non-full leaf $l$, we set alloc_rates $l_{l}$ (which is simply a scalar) to be 1 , alloc_bound ${ }_{l}$ to be the remaining capacity of $l$, and $\max _{-} v u l_{l}(x)$ to be $(P(l)+x) / w_{l}$. Note that at the start of the first iteration, $P(l)=0$, so we have invariant (I2) hold trivially. 
Recursive computation of alloc_rates $_{j}$, alloc_bound ${ }_{j}$, and $m a x \_v u l_{j}$. Fix a non-full internal node $j$ and let $H_{j}$ denote the set of non-full children of $j$. Note that since $j$ is non-full, $H_{j}$ is not empty.

We first describe how to compute alloc_rates ${ }_{j}$, while also establishing invariant (I2) for the start of the next iteration. By invariant (I2), max_vul $i_{i}(0)$ is the same for all non-full child $i$ of $j$. Consider equating max_vul $l_{i}\left(x_{i}\right)$ with $\max _{-} v u l_{k}\left(x_{k}\right)$ for all non-full children $i$ and $k$ in $H_{j}$. Since these equations are linear, we can obtain a solution to the set of equations such that $x_{k}$ for each $k$ is a scalar multiple of $x_{i}$. Note that these equations ensure that if the number of additional tasks assigned to the subtree rooted at $k$ in this iteration is proportional to $x_{k}$, for each non-full child $k$ of $j$, then at the start of the next iteration, $\max _{-} v u l_{k}(0)$ will be the same for each non-full child $k$ of $j$. We now set alloc_rates $j_{j}$ to be the vector obtained by putting together $x_{k} \cdot$ alloc_rates $_{k}$, over all non-full children $k$ of $j$, and the all-zeros vector for each full child of $j$, scaled so that the sum of the values in the vector equals 1 . This particular choice of vector alloc_rates $j_{j}$ ensures that the vulnerability for all leaves within $T_{j}$ that have no full ancestors in $T_{j}$ remains the same when additional tasks are assigned according to the proportions given by alloc_rates $j_{j}$. Thus, invariant (I2) continues to hold at the start of the next iteration.

We next describe the computation of $\max _{-} v u l_{j}$. Fix an arbitrary node $i$ in $H_{j}$. If $P(j) / w_{j}>$ $\max \_v l_{i}(0)$, then this implies that the contribution of $j$ to the vulnerability calculation of Equation (1) exceeds that of any node inside the subtree rooted at each of the non-full children of $j$. We set $\max _{-} v u l_{j}(x)$ to be $(P(j)+x) / w_{j}$. If $P(j) / w_{j} \leq \max _{-} v u l_{i}(0)$, then the vulnerability of every leaf in $L(j)$ that has no full ancestor in the subtree rooted at $j$ is the same: $\max _{-} v u l_{i}(0)$. When $x$ units of tasks are added to the subtree rooted at $j$, then the number of tasks added to the subtree rooted at $i$ equals $x \cdot\left(\sum_{l \in T_{i}}\right.$ alloc_rates $\left._{j}(l)\right)$. Therefore, for any leaf $l$ in $L(j)$, the maximum vulnerability of a leaf in $T_{j}$ varies with $x$ as $\max _{-} v u l_{j}(x)=\max _{-} v u l_{i}\left(x \cdot\left(\sum_{l \in T_{i}}{\text { alloc } \text { rates }_{j}}(l)\right)\right)$.

We finally describe the computation of alloc_bound $d_{j}$. It is helpful for us to define an auxiliary parameter $u_{i}$. If $P(j) / w_{j}>\max _{-} v u l_{i}(0)$, then we define $u_{i}$ to be the minimum of alloc_bound ${ }_{i}$ and the smallest $x_{i}$ such that $\left(P(j)+x_{i}\right) / w_{j} \leq$ max_vul $l_{i}\left(x_{i}\right)$ (the point at which the ratios that contribute to the vulnerability calculation switch); otherwise, $u_{i}$ is set to alloc_bound $d_{i}$. Finally, we set alloc_bound $d_{j}$ to be the minimum of the remaining capacity at $j$ and the minimum, over all $k \in H_{j}$, of $u_{k} /\left(\sum_{l \in T_{k}}\right.$ alloc_rates $\left._{j}(l)\right)$.

Updating the placement. Using the above computation in a bottom-up manner, we compute for the root node $t$ : alloc_rates , alloc_bound $_{t}$, and max_vul $(x)$. We set $P$ to be the new allocation given by the vector sum $P+$ alloc_rates $_{t} \cdot \min \left\{\right.$ alloc_bound $\left._{t}, N-|P|\right\}$, where we use $|P|$ here to denote the number of tasks allocated in $P$.

The generalized spreading algorithm repeats the above process until the required number of tasks have been allocated.

THEOREM 4.6. The generalized spreading algorithm computes a fractional placement that is simultaneously optimal for all $W$ for HIERFRACROBUSTFAP, in time polynomial in the size of the input.

Proof. We first show that the generalized spreading algorithm computes a fair placement, by establishing invariant (I1). The proof is by induction on the number of iterations. As described above in the algorithm, invariant (I1) holds at the start of the algorithm; so the induction base holds. For the induction step, we assume that invariant (I1) holds at the start of iteration $\eta \geq 1$, and we aim to establish the invariant at the start of the iteration $\eta+1$. Let $P$ denote the placement at the start of the iteration $\eta+1$. Let $T$ be any subtree rooted at node $j$. Let $l$ and $l^{\prime}$ be two leaf nodes in a subtree $T$ rooted at a node $j$ such that $p_{l}(T, P)<p_{l^{\prime}}(T, P)$ at the start of iteration $\eta+1$. We show that $l$ must have a full ancestor in $T$. The proof is by contradiction. If all ancestors of $l$ in $T$ are nonfull, then let $i$ and $k$ be the children of $j$ that are ancestors of $l$ and $l^{\prime}$, respectively. (Note that $i$ may 
be equal to $k$.) Note that $i$ is not full by our assumption. If $k$ was full at the start of iteration $\eta$, then no new tasks are added to the subtree rooted at $k$ in iteration $\eta$. This implies that either $p_{l^{\prime}}(T, P)$ has not changed since the last iteration or $p_{l^{\prime}}(T, P)=P(j) / w_{j}$. In the former case, since $p_{l}(T, P)$ can never decrease, invariant (I1) must have been false at the start of iteration $\eta$, contradicting the induction hypothesis. In the latter case, $p_{l}(T, P) \geq P(j) / w_{j}=p_{l^{\prime}}(T, P)$, contradicting our assumed relationship between $p_{l}(T, P)$ and $p_{l^{\prime}}(T, P)$.

We thus assume that $k$ was also not full at the start of iteration $\eta$. By the definitions of vulnerability (Definition 4.1) and max_vul, we obtain that $p_{l}(T, P)$ (resp., $p_{l^{\prime}}(T, P)$ ) is the maximum of $\max _{-} v u l_{i}(0)\left(\right.$ resp., $\left.m a x_{-} v u l_{k}(0)\right)$ and $P(j) / w_{j}$. By invariant (I2), max_vul $l_{i}(0)$ equals max_vul $l_{k}(0)$ at the start of iteration $\eta+1$. We thus have $p_{l}(T, P)=p_{l^{\prime}}(T, P)$, contradicting our assumption. This establishes the fairness of the placement at the start of iteration $\eta+1$, thus completing the induction step. We have thus established that the algorithm computes a fair placement. Combining this claim with Lemmas 4.5 and 4.4 yields the optimality of the placement returned by the algorithm.

We now show that the algorithm completes in polynomial time. In each iteration, either a node reaches capacity or a new node achieves the minimum vulnerability bound. Thus, the number of iterations where a new node reaches capacity is at most the number of nodes. And between two such iterations, each iteration has a new node achieving the minimum vulnerability bound. This leads to an upper bound of $n^{2}$ on the number of iterations, where $n$ is the number of nodes. The running time of each iteration is linear in the size of hierarchy, leading to a polynomial time bound on the running time of the algorithm.

\subsection{Randomized Algorithm for HierRobustFAP}

Though the generalized spreading algorthm is inherently "continuous," it suggests natural algorithms for HierRobustFAP. One discrete version of generalized spreading for HierRobUSTFAP is to execute the same high-level incremental procedure, but allocating units of tasks in each iteration, as opposed to fractions. An analysis of this algorithm is an open problem. We now present a basic randomized rounding of generalized spreading that offers performance guarantees against oblivious adversaries.

Suppose $P$ is the fractional placement returned by generalized spreading. We number the machines in the hierarchy in order (say, left to right in appearance in the domain tree) so that all the machines belonging to a domain appear contiguously. With machine $i$, we associate the interval $I(i)=\left[\sum_{j=1}^{i-1} P(j), \sum_{j=1}^{i} P(j)\right)$. We select a number $\rho$ uniformly at random from $[0,1]$. Consider the set $K=\{k+\rho: k \in \mathbb{Z}\}$. In our solution, the number of tasks we place in machine $i$ equals the size of the intersection $I(i) \cap K$.

We now analyze the guarantee provided by the above randomized algorithm. For any machine $i$, the expected number of tasks placed on $i$ equals the number of tasks $P(i)$ since it exactly equals the length of the interval $I(i)$. By linearity of expectation, the expected number of tasks placed on any set $S$ of domains is $P(S)$. Therefore, for any weight bound $W$, if the optimal solution to HierFracRobustFAP achieves an objective of $A_{W}^{*}$, then for any subset $S$ of domains of weight at most $W$, the expected number of tasks placed by the randomized algorithm on the domains in $S$ is at most $A_{W}^{*}$. Furthermore, for any domain, the number of tasks placed on the domain is within one of that placed by the optimal fractional solution. This yields the following lemma.

Lemma 4.7. For any weight bound $W$, let $A_{W}^{*}$ denote the objective value achieved by the optimal solution to HIERFRACROBUSTFAP. There exists a randomized algorithm for computing a placement for HIERROBUSTFAP such that for any $W$ and any subset $S$ of domains of total weight $W$, the expected number of tasks placed in $S$ is at most $A_{W}^{*}$. 


\subsection{Proofs for Lemmas 4.4 and 4.5}

We start with characterizing an optimal adversary. The adversary's maximization problem for a given placement $P$ is given in Linear Program (2) below, where $P(u)$ denotes the number of tasks placed by $P$ in the subtree rooted at $u$.

$$
\begin{aligned}
A(P) & =\max \sum_{u} x_{u} P(u) \\
\sum_{u} x_{u} w_{u} & \leq W \\
\sum_{u \in \operatorname{anc}(v)} x_{u} & \leq 1 \text { for every machine } v \\
x_{u} & \leq 1 \text { for every domain } u \\
x_{u} & \geq 0 \text { for every domain } u
\end{aligned}
$$

The following two lemmas provide insight into a solution to the adversary's maximization problem.

LeMmA 4.8. Given any placement $P$, there exists an optimal solution $x$ for the adversary in which there exists at most one node $v$ such that $x_{v}$ is not 0 and the sum of $x_{u}$, over all ancestors $u$ of $v$, is in $(0,1)$.

Proof. Let $x$ be an optimal extreme point solution to the LP (an optimal vertex of the associated polytope). For any $u$, if $x_{u}$ is 0 , we remove $x_{u}$ from the LP and $u$ from the hierarchy; for any $x_{u}$ that is 1 , we add $P(u)$ as a constant into the LP objective and constraints, and remove the subtree rooted under $u$. We thus have an LP with number of variables equal to the number of nodes in the resulting forest, and the number of constraints equal to one more than twice the number of nodes. Since the number of tight constraints in an extreme point solution equals the number of variables and none of the range constraints are tight, it follows that all but at most one of the sum constraints are tight, thus completing the proof of the desired claim.

Lemma 4.9. Given any placement $P$, there exists an optimal solution $x$ for the adversary in which there exists at most one node $v$ such that $x_{v}$ is in $(0,1)$.

Proof. By Lemma 4.8, there exists an optimal solution $x$ for the adversary in which there is at most one node $v$ such that $x_{v}$ is not 0 and the sum of $x_{u}$, over all ancestors $u$ of $v$, is in $(0,1)$. We refer to such as node $v$, if it exists, as an outlier. Let $S$ be the set of nodes such that for each $s$ in $S, x_{s} \in(0,1)$. Fix an $s$ in $S$. If there exists an ancestor $s^{\prime}$ of $s$ such that $x_{s^{\prime}}$ is non-zero, then $s^{\prime}$ is an outlier; otherwise, $s$ is an outlier. Given that there is at most one outlier, if $|S|>1$, then there exists a unique node $t$ in $S$ such that (a) $t$ is an outlier and an ancestor for all nodes in $S$, (b) no node in $S-t$ is an ancestor of any other node in $S-t$, and (c) for every $s$ in $S-t, x_{s}$ equals $1-x_{t}$.

We now argue that $x$ can be transformed into another optimal solution $x^{\prime}$ for the adversary, which satisfies the claim of the corollary. We consider three cases. In the first case, $w_{t}<w(S-t)$, where $w(S-t)$ is the sum of the weights of the nodes in $S-t$. In this case, we set $x_{t}^{\prime}$ to be $x_{t}+\varepsilon$ and $x_{s}^{\prime}$ to be $x_{s}-\varepsilon^{\prime}$, where $\varepsilon>\varepsilon^{\prime}>0$ is a real number made sufficiently small. The weight constraint is maintained by the adversary by making $\varepsilon^{\prime}$ and $\varepsilon$ sufficiently small, while the objective values increase, yielding a contradiction. In the second case, we have $w_{t}>w(S-t)$. Let $\varepsilon_{t}=\delta / w_{t}$ and $\varepsilon_{s}=\delta / w(S-t)$. If $P(t) / w_{t} \geq P(S-t) / w(S-t)$, then we set $x_{t}^{\prime}=x_{t}+\varepsilon_{t}$ and $x_{s}^{\prime}=x_{s}-\varepsilon_{s}$ for all $s \in S-t$. Otherwise, we set $x_{t}^{\prime}=x_{t}-\varepsilon_{t}$ and $x_{s}^{\prime}=x_{s}+\varepsilon_{s}$ for all $s \in S-t$. By the choice of $\varepsilon_{s}$ and $\varepsilon_{t}$, the weight constraint for the adversary is satisfied. And in either of the two subcases, the objective value of the adversary improves, yielding a contradiction. 
We are finally left with the case $w_{t}=w(S-t)$. In this case, we set $x_{t}=1$ and $x_{s}=0$ for all $s \in$ $S-t$. Clearly, the weight constraint is satisfied, and the objective value of the adversary remains the same, establishing the desired claim of the corollary.

For any leaf $u$ and subtree $T$ containing $u$, and knapsack solution $x$ to the adversarial maximization problem, let $y_{u}(T, x)$ denote the sum, over all ancestors $v$ of $u$ in $T$, of $x_{v}$.

Lemma 4.10. Given any placement $P$ and any weight bound $W$, in every optimal solution $x$ to the adversary's maximization problem, for any two leaf nodes $i$ and $j$ and any subtree $T$ containing $i$ and $j$, if $p_{i}(T, P)>p_{j}(T, P)$, then $y_{i}(T, x) \geq y_{j}(T, x)$.

Proof. The proof is by contradiction. Let $i$ and $j$ be two leaves and $T$ be a subtree containing them such that $p_{i}(T, P)>p_{j}(T, P)$ and $y_{i}(T, x)<y_{j}(T, x)$. Since $y_{i}(T, x)<y_{j}(T, x)$, there must exist an ancestor $r$ of $j$ in $T$ such that $x_{r}>0$. Let $q$ denote the ancestor of $i$ that is the argmax of $\max _{\text {ancestor } l \text { of } i \text { in } T} P(l) / w_{l}$. Then, we have $P(q) / w_{q}=p_{i}(T, P)>p_{j}(T, P) \geq P(r) / w_{r}$. We also have $x_{q} \leq y_{i}(T, x)<y_{j}(T, x) \leq 1$. Consider an alternative adversarial solution $x^{\prime}$ that is identical to $x$ except that $x_{r}^{\prime}=x_{r}-\varepsilon, x_{q}^{\prime}=x_{q}+w_{r} / w_{q}$, for $\varepsilon \leq \min \left\{1-x_{q}, x_{r}\right\}$. The difference between the total value of the knapsack $x^{\prime}$ and that of $x$ equals $\varepsilon w_{r} k_{q} / w_{q}-\varepsilon k_{r}$, which is positive since $k_{q} / w_{q}>k_{r} / w_{r}$. This contradicts the assumption that $x$ is optimal, thus completing the proof of the lemma.

Proof of Lemma 4.4: Let $P$ be an optimal (fractional) placement for the weight bound $W$. Suppose, for the sake of contradiction, that there does not exist any optimal hierarchical max-min fair allocation. We consider any linear order $<$ of all of the subtrees of the hierarchy in which $T_{1}<T_{2}$ whenever $T_{1}$ is a subtree of $T_{2}$. For any allocation $P$, let $\operatorname{muf}(P)$ denote the minimum subtree, according to this linear order, such that $P$ is not hierarchical max-min fair within that subtree. (The name "muf" stands for "minimum unfair" subtree.) By our assumption, such a subtree exists. Let lvs $(P)$ denote the number of leaves $i$ in $\operatorname{muf}(P)$ such that $p_{i}(\operatorname{muf}(P), P)$ equals $\max _{j \in \operatorname{muf}(P)} p_{j}(\operatorname{muf}(P), P)$.

Let $P$ denote an optimal allocation, which is lexicographically minimum with respect to the triple

$$
\left(\operatorname{muf}(P), \max _{j \in \operatorname{muf}(P)} p_{j}(\operatorname{muf}(P), P), \operatorname{lvs}(P)\right) .
$$

Let $T$ equal muf $(P)$. We thus have two leaf nodes $i$ and $j$ of $T$ such that $j$ has the maximum vulnerability in $T$ and $p_{i}(T, P)<p_{j}(T, P)$, and yet every ancestor of $i$ in $T$ is not full. We argue now by contradiction that $i$ and $j$ belong to two different proper subtrees of $T$. If $i$ and $j$ belong to the same proper subtree, say $T^{\prime}$, of $T$, then since $P$ is hierarchical max-min fair within $T^{\prime}, p_{i}\left(T^{\prime}, P\right) \geq p_{j}\left(T^{\prime}, P\right)$, which also implies that $p_{i}(T, P) \geq p_{j}(T, P)$ leading to a contradiction.

Let $i$ and $j$ belong to proper subtrees rooted at, say, $u$ and $v$, respectively; we refer to these trees as $T_{u}$ and $T_{v}$, respectively. By our assumption, the allocations within both $T_{u}$ and $T_{v}$ are hierarchical max-min fair. Consider an alternative allocation $P^{\prime}$ that is identical to $k$ except that an $\varepsilon>0$ amount of tasks are added to the tree rooted at $u$, and an amount decreased from the tree rooted at $v$. We do this following the algorithm defined above; this is feasible since the allocation $P$, restricted to $u$ (or $v$ ), is hierarchical max-min fair, and hence, produced by the algorithm (following our assumption about $T$ being $\operatorname{muf}(P))$. The amount is chosen sufficiently small such that $p_{i}\left(T, P^{\prime}\right)<p_{j}\left(T, P^{\prime}\right)$, and every non-full node in subtree rooted at $u$ maintains its capacity constraints, and $P^{\prime}(l) \geq 0$ for all $l$ in the tree rooted at $v$. Clearly, $P^{\prime}$ is a valid allocation. Furthermore, since $j$ has maximum vulnerability in $P, P^{\prime}$ has either a smaller maximum vulnerability than $P$ or $P^{\prime}$ has fewer leaf nodes with the maximum vulnerability value. In either case, $P^{\prime}$ is lexicographically smaller with respect to the metric given above. We now derive a contradiction by arguing that $P^{\prime}$ is also an optimal allocation. 
Let $s^{\prime}$ be an optimal knapsack of weight $W$ and value $v^{\prime}$ built from $P^{\prime}$. Note that $j$ has the largest vulnerability in the tree rooted at $v$ and $i$ has the largest vulnerability in the tree rooted at $u$. Let $L_{v}$ denote the set of leaves in the tree rooted at $v$ that have the largest vulnerability among all leaves in the tree rooted at $v$. Similarly, let $L_{u}$ denote the set of leaves in the tree rooted at $v$ that have the largest vulnerability among all leaves in the tree rooted at $v$. Note that $j \in L_{v}$ and $i \in L_{u}$. Since $j$ has higher vulnerability than $i$, we obtain from Lemma 4.10 that $s^{\prime}$ will select at least as much fraction of any leaf in $L_{v}$ as that of any leaf in $L_{u}$. Since the amount of the former is less in $P^{\prime}$ than in $P$ by $\varepsilon$, while that of the latter is more in $P^{\prime}$ than in $P$ by $\varepsilon$, we obtain that the value of the knapsack $s^{\prime}$ against allocation $P$ is at least that of $s^{\prime}$ against $P^{\prime}$. Thus, for any weight $W$, an optimal weight- $W$ knapsack against $P$ has at least as much value as an optimal weight- $W$ knapsack against $P^{\prime}$. Hence, $P^{\prime}$ is optimal and lexicographically smaller than $P$, contradicting our assumption about $P$. This completes the proof of the lemma.

Proof of Lemma 4.5: The proof is by induction on the height of the hierarchy. For the induction basis (hierarchies of height 1), the claim follows from the single-level case. For the induction step, consider any hierarchy of height $h$. Our proof is by contradiction. For a given number $N$, let, if possible, there be two distinct hierarchical max-min fair solutions $P$ and $P^{\prime}$. Since the claim is true for hierarchies of height smaller than $h$, it follows that there exist two children $i$ and $j$ of the root such that $P(i)>P^{\prime}(i)$ while $P(j)<P^{\prime}(j)$. Suppose $T_{i}$ and $T_{j}$ denote the subtrees rooted under $i$ and $j$, respectively. Then, there exists a leaf node $l$ in $T_{i}$ such that every ancestor of $l$ in $T_{i}$ is not full in $P$, and a leaf node $l^{\prime}$ in $T_{j}$ such that every ancestor of $l^{\prime}$ in $T_{j}$ is not full in $P^{\prime}$. We thus have $p_{l}\left(T_{i}, P\right) \geq p_{l^{\prime}}\left(T_{j}, P^{\prime}\right)$ and $p_{l}\left(T_{i}, P^{\prime}\right) \leq p_{l^{\prime}}\left(T_{j}, P\right)$. But since $P(j)<P^{\prime}(j)$ and $P(i)>P^{\prime}(i)$, and the unique hierarchical max-min fair allocations for the two hierarchies rooted at $i$ and $j$, respectively, have the property that the vulnerability of an non-full leaf node only increases with the number of tasks, we have $p_{l}\left(T_{i}, P^{\prime}\right)>p_{l}\left(T_{i}, P\right)$ and $p_{l^{\prime}}\left(T_{j}, P\right)>p_{l^{\prime}}\left(T_{j}, P^{\prime}\right)$. These inequalities yield $p_{l}\left(T_{i}, P^{\prime}\right)>$ $p_{l^{\prime}}\left(T_{j}, P^{\prime}\right)$, implying that there exists a full ancestor of $\ell^{\prime}$ in $P^{\prime}$, yielding a contradiction, completing the induction step and the proof of the lemma.

\section{PROBABILISTIC FAILURES: BICRITERIA APPROXIMATIONS}

We now present bicriteria polynomial-time approximations for ProbFAP and HierProbFAP. In particular, our algorithm for PROBFAP achieves an $\varepsilon$ additive approximation in the failure probability, while giving up a $(1+\varepsilon)$ multiplicative factor in the number of failures. We next extend the result to hierarchies by giving an algorithm for HIERPROBFAP, which achieves an $\varepsilon$ additive approximation in failure probability, while giving up a $(L+\varepsilon)$ multiplicative factor in the number of failures, for $L$-level hierarchies, where $L$ is a constant.

\subsection{Single-Level Case: ProbFAP}

Our solution approach here builds on the stochastic optimization framework of Li and Yuan (2013). For each domain $i$ and integer $b$ in $\left[0, C_{i}\right]$, we introduce an item $(i, b)$ with associated random variable $X(i, b)$, capturing the fraction of failed tasks if domain $i$ fails, that has the following distribution:

$$
X(i, b)= \begin{cases}0 & \text { with probability } 1-p_{i} \\ \frac{b}{N} & \text { with probability } p_{i}\end{cases}
$$

Define $U$ as the universe of all items; i.e., $U=\left\{(i, b): i \in \mathcal{F}, 0 \leq b \leq C_{i}\right\}$. We define a subset $S$ of $U$ as feasible if (a) for each $i \in \mathcal{F}$, there is exactly one item of the form $(i, b)$ in $S$; and (b) $\sum_{(i, b) \in S} b=N$.

For a feasible solution $S$, define $X(S)=\sum_{(i, b) \in S} X(i, b)$. ProbFAP is then exactly the threshold probability maximization problem where we aim to maximize $\operatorname{Pr}[X(S) \leq F / N]$. Li and Yuan show 
that a PTAS for an associated multi-dimensional exact version of the problem implies an approximation scheme for the threshold probability maximization problem. Their framework, however, assumes that the random variables $X()$ are mutually independent. This is not true for our problem, since while $X(i, b)$ is independent of $X\left(j, b^{\prime}\right)$ for any $i \neq j, b, b^{\prime}$, the variables $X(i, b)$ and $X\left(i, b^{\prime}\right)$ are not independent of each other. Fortunately, in our problem, any feasible set of items consists of at most one item of the form $(i, b)$ for any given $i$; so, the random variables associated with the items in any feasible set are all mutually independent. We use this fact to extend the Li-Yuan framework for the single-level case.

We now present the mechanism of classifying items as light or heavy, discretizing the size distributions of light items, enumerating all possible heavy item sets, and signatures of light item sets.

Item classification. Call an item $(i, b)$ light if $E[X(i, b)] \leq \varepsilon^{10}$, and heavy otherwise.

Discretization. For item $(i, b)$, we define variable $\tilde{X}(i, b)$ that takes values in $\left\{s_{j}=j \varepsilon^{5}: 0 \leq j \leq\right.$ $\left.\left\lfloor 1 / \varepsilon^{5}\right\rfloor\right\}$. We map $X(i, b)$ to $\tilde{X}(i, b)$ as follows. If $X(i, b)>\varepsilon^{4}$, then $\tilde{X}(i, b)=\left\lfloor X(i, b) / \varepsilon^{5}\right\rfloor \varepsilon^{5}$. For $X(i, b) \leq \varepsilon^{4}$, we find a $d$ such that $\varepsilon^{4} \cdot \operatorname{Pr}\left[X(i, b) \geq d \mid X(i, b) \leq \varepsilon^{4}\right]=E\left[X(i, b) \mid X(i, b) \leq \varepsilon^{4}\right]$, and set $\tilde{X}(i, b)=0$ for $0 \leq X(i, b)<d$ and $\tilde{X}(i, b)=\varepsilon^{4}$ for $d \leq X(i, b) \leq \varepsilon^{4}$. It is easy to show that for any feasible set of items, the distribution of the sum of the discretized variables for the set is close to that for the original variables.

Lemma 5.1 ((Li And Yuan 2013, Lemma 2.2)). For any set $S$ of items such that $E[X(S)] \leq 3 / \varepsilon$, for any $k$, we have

$$
|\operatorname{Pr}[X(S) \leq k]-\operatorname{Pr}[\tilde{X}(S) \leq k]|=O(\varepsilon) .
$$

Enumeration of heavy item sets. We enumerate all possible heavy item sets $H$ with $E[X(H)]<$ $3 / \varepsilon$. Each heavy item set is of constant cardinality; hence, the number of such sets is polynomial. In the remainder, we can assume a fixed heavy item set $H$.

We now introduce a notion of signatures for light item sets.

Definition 5.2. For an item $(i, b)$, we define its signature to be the vector $\left(\pi_{i, b}\left(s_{1}\right), \ldots, \pi_{i, b}\left(s_{z-1}\right)\right)$, where $z=\left\lfloor 1 / \varepsilon^{5}\right\rfloor$ and

$$
\pi_{i, b}(s)=\frac{\varepsilon^{6}}{n}\left\lfloor\frac{n}{\varepsilon^{6}} \cdot \operatorname{Pr}[\tilde{X}(i, b)=s]\right\rfloor
$$

for all $s$ in $\left\{s_{1}, s_{2}, \ldots, s_{z-1}\right\}$. We define the signature of a set of items to be the coordinate-wise sum of the signatures of the individual items in the set.

A key claim (Li and Yuan 2013, Lemma 2.3) shows that it is sufficient to consider signatures whose components add up to at most $3 / \varepsilon$, and enumerate signatures for a set of light items. The proof of this lemma relies on the Poisson approximation theorem for sums of independent random variables (Le Cam 1960). Since we apply the Possion approximation theorem for random variables associated with the items in any feasible set, the independence of these variables continues to hold, and so the same lemma holds.

LEMMA 5.3. Let $S_{1}$ and $S_{2}$ be two sets of light items, with the same signature, and $E\left[\tilde{X}\left(S_{1}\right)\right] \leq 3 / \varepsilon$, $E\left[\tilde{X}\left(S_{2}\right)\right] \leq 3 / \varepsilon$. Then, the total variation distance between $X\left(S_{1}\right)$ and $X\left(S_{2}\right)$ is $O(\varepsilon)$.

Using Lemmas 5.1 and 5.3, we then obtain the following equivalent of Theorem $1.1 \mathrm{of} \mathrm{Li}$ and Yuan (2013). Suppose there is a pseudopolynomial algorithm for an exact version of ProBFAP, that is, the problem of determining whether, for a given weight bound $\mathcal{W}$, there is a feasible solution of weight $\mathcal{W}$ for a given instance of ProbFAP where individual elements have weights. Then there is a polynomial-time approximation algorithm for ProBFAP that finds a feasible solution 
$S$ such that $\operatorname{Pr}[X(S) \leq(1+\varepsilon) F / N] \geq \mathrm{OPT}-\varepsilon$, where OPT is the maximum value achievable for $\operatorname{Pr}[X(S) \leq F / N]$, for any feasible $S$.

Algorithm for an exact version of ProbFAP. In the exact version of ProbFAP, each element $(i, b)$ of $U$ is associated with a weight $\omega_{i, b}$, where the weight will represent the item signature we have defined above in Definition 5.2. We are given a weight constraint $W$ and we would like to determine if there is a feasible solution $S$ such that $\sum_{(i, b) \in S} \omega_{i, b}=\mathcal{W}$. Note that $\mathcal{W}$ represents the overall signature we desire for the set of light items. We define $\Pi(X, M, j)$ to be a predicate that is true if it is possible to assign tasks to domains 1 through $j$ so that the total weight of the assignment is exactly $X$. We set up a dynamic program as follows: $\Pi(0,0,0)$ is true; $\Pi(X, M, j)$ is true if $\Pi(X-w(i, b), M-b, j-1)$ is true for any $0 \leq b \leq C_{j}$, and false otherwise. It can be seen that the above can be computed in time polynomial in the number of tasks $N$, the number of possible weight values, and the number of domains $n$. Furthermore, the algorithm extends to the scenario where the weights are constant-dimensional vectors, as is the case with the signatures; the exponent in the polynomial time grows with the dimension of the weight vectors. Finally, note that our algorithm as presented is polynomial in $N$, not the size of $N$. We apply the standard method of restricting $b$ values in the items $(i, b)$ to rounded powers of $(1+\varepsilon)$. This causes us another factor of $(1+\varepsilon)$ loss in the number of failed tasks, and yields the following single-level special case of Theorem 1.10.

THeOREM 5.4. For any $\varepsilon>0$, there exists a polynomial time algorithm that runs in time $n^{\text {poly }(1 / \varepsilon)}$ and returns a feasible placement of $N$ tasks such that

$$
\operatorname{Pr}[\text { number of failed tasks } \leq(1+\varepsilon) F] \geq \mathrm{OPT}-\varepsilon,
$$

where OPT is the maximum probability, over any placement of $N$ tasks, that the number of failed tasks is at most $F$.

\subsection{Hierarchical Case: HierProbFAP}

We next consider the hierarchical version, HierProbFAP. For each node $i$, we define an item $(i, b)$ for each $0 \leq b \leq C_{i}$. As before, we have the associated random variable $X(i, b)$, that takes value 0 with the probability that at least one of its ancestor domains fails, and $b / N$ otherwise. The $X(i, b)$ variables are no longer independent, even for different $i$. This is because nodes share ancestor domains.

One approach to this problem is to solve multiple stochastic knapsack problems, one for each of the $L$ levels, using the single-level algorithm of Section 5.1 and then select the best of all the solutions. This approach is unlikely to yield any useful approximation guarantees, however, since the solution for any one level may perform poorly for other levels. We present an approximation algorithm that considers the multiple level problems in an integrated manner and identifies a single solution that performs close to the optimal at each level. Let $P_{\ell}(E)$ denote the probability of event $E$, over the failure events of domains at level $\ell$; that is,

$$
P_{\ell}(E)=\operatorname{Pr}[E \mid \text { no domain at any level } i \neq \ell \text { fails }] .
$$

For subproblem at level $\ell$, we enumerate a polynomial number of solutions to find all feasible solutions $S_{\ell}$ such that $P_{\ell}\left(X\left(S_{\ell}\right) \leq(1+\varepsilon / L) F / N\right) \leq \mathrm{OPT}_{\ell}-\varepsilon / L$, where $\mathrm{OPT}_{\ell}$ is the maximum probability, over any placement of $N$ tasks, that the number of failed tasks due to failures exclusively at level $\ell$ is at most $F$. By our enumeration of the solutions, we will identify a single solution $S$ such that $P_{\ell}(X(S) \leq(1+\varepsilon) F / N) \geq \mathrm{OPT}_{\ell}-\varepsilon / L$ for each $\ell$. By combining the bound over all the levels, we will establish that $S$ satisfies

$$
\operatorname{Pr}[X(S) \leq(L+\varepsilon) F / N] \geq \mathrm{OPT}-\varepsilon .
$$


For each level, as in Section 5.1, we classify items as light or heavy based on their expected size, discretize the size distributions for light items, and enumerate all possible heavy item sets and signatures for light item sets. The item classification and discretization is the same as in Section 5.1. The heavy item sets and the dynamic program we set up to process the light item sets across all levels in an integrated manner are described below.

Enumeration of heavy item sets. We enumerate all possible heavy item sets $H$ with $E[X(H)]<$ $3 / \varepsilon$ at each level. Each heavy item set is of constant cardinality, hence, the number of distinct heavy sets at each level is polynomial. Since the number of levels is constant, there is a polynomial number of ways to select a heavy item set at each level.

Dynamic programming for processing the light item sets. Fix a heavy item set $H_{\ell}$ for each level $\ell$ of the hierarchy. Fix a signature $\sigma_{\ell}$ for each level $\ell$ of the hierarchy. Recall from Definition 5.2 that each signature is a vector with $O\left(\varepsilon^{-5}\right)$ coordinates, and the value of each coordinate is bounded by $O(n)$.

Our goal now is to determine whether there is a selection of items so that $L_{\ell}$ is the set of light items selected at level $\ell, H_{\ell} \cup L_{\ell}$ is feasible for each $\ell$, and $S g\left(L_{\ell}\right)=\sigma_{\ell}$ for each $\ell$; here, $S g(I)$ of a set $I$ of light items denotes the signature of the set. We set up a dynamic program that determines if there is a feasible selection of light items satisfying the preceding condition. For this purpose, we define a predicate $\Phi$ as follows. Let $\lambda(v)$ denote the level of node $v$ in the hierarchy. For a given node $v$, positive integer $i$ at most the number of children of $v$, and sequence of signature vectors $\sigma_{1}, \sigma_{2}, \ldots, \sigma_{\lambda(v)}$, we define $\Phi\left(v, i, \sigma_{1}, \ldots, \sigma_{\lambda(v)}\right)$ to be true if there exists a feasible selection of light items for the subtree consisting of $v$, the first $i$ children of $v$, and their descendants such that the signature of the set of light items at level $\ell, 1 \leq \ell \leq \lambda(v)$, equals $\sigma_{\ell}$. We compute $\Phi()$ using the following dynamic program. For a leaf node $v$, we set

$$
\Phi(v, 0, \sigma)=\left\{\begin{array}{l}
\text { true if } \sigma=(0,0, \ldots) \text { or } \exists b: S g(\{(v, b)\})=\sigma \\
\text { false otherwise. }
\end{array}\right.
$$

For an internal node $v$, let $d(v)$ denote the number of children of $v$, and let $v_{i}$ denote the $i$ th child of $v$, numbered in an arbitrary order. For internal node $v$, we compute that for $i>0$, $\Phi\left(v, i, \sigma_{1}, \ldots, \sigma_{\lambda(v)}\right)$ equals

$$
\begin{gathered}
\bigvee_{\sigma_{1}^{\prime}, \ldots, \sigma_{\lambda(v)-1}^{\prime}}\left(\Phi\left(v, i-1, \sigma_{1}-\sigma_{1}^{\prime}, \ldots, \sigma_{\lambda(v)-1}-\sigma_{\lambda(v)-1}^{\prime}, \sigma_{\lambda(v)}\right)\right. \\
\left.\wedge \Phi\left(v_{i}, d\left(v_{i}\right), \sigma_{1}^{\prime}, \ldots, \sigma_{\lambda(v)-1}^{\prime}\right)\right),
\end{gathered}
$$

where we note that $\lambda\left(v_{i}\right)=\lambda(v)-1$. Finally, $\Phi\left(v, 0, \sigma_{1}, \ldots, \sigma_{\lambda(v)}\right)$ is exactly the single-level problem for node $v$ in which we determine if there exists a selection of light items for the domains $v_{1}$ through $v_{d(v)}$ such that the heavy items selected for this subset of domains, together with the light items, form a feasible solution for this set of domains, and the signature of the light item set matches $\sigma_{\lambda(v)}$.

Final output. We have written the above dynamic program as a predicate; it is straightforward to extract an actual solution that satisfies the condition desired in $\Phi$. Thus, after solving the dynamic program, for any combination of signatures $\sigma_{i}$ at level $i$, for $i \leq \ell$, if there exists a solution that has the signature $\sigma_{i}$ at level $i$, for $i \leq \ell$, we obtain one such solution. Among these, we select one solution that has the highest product $\prod_{l} P_{\ell}\left(X_{\ell}(S) \leq(1+\varepsilon) F / N\right)$, which we can estimate using the signatures, as in the single-level subproblem.

Analysis of the algorithm. Since the number of different signature values is polynomial $\left(n^{O\left(\varepsilon^{-5}\right)}\right)$ and the number of levels is a constant, the total number of different inputs for which $\Phi$ is being 
computed is polynomial in $n$. This ensures that in polynomial time, we determine a feasible solution $S$ of items such that at each level $\ell$, the signature of $S$ matches that of a globally optimal solution, say $S^{*}$. If $X_{\ell}(S)$ (resp., $X_{\ell}\left(S^{*}\right)$ denotes the number of failed tasks in $S$ (resp., $S^{*}$ ) due to failures in level $\ell$, then Lemma 5.3 implies that at each level $\ell$ the variation distance between $\tilde{X}_{\ell}(S)$ and $\tilde{X}_{\ell}\left(S^{*}\right)$ is $O(\varepsilon)$. Together with Lemma 5.1, we get

$$
P_{\ell}\left(X_{\ell}(S) \leq(1+\varepsilon) F / N\right) \geq \mathrm{OPT}_{\ell}-O(\varepsilon),
$$

where $\mathrm{OPT}_{\ell}$ is the maximum probability, over any placement of $N$ tasks, that the number of failed tasks due to failures exclusively at level $\ell$ is at most $F$. Since the failure events at each of the failure domains are independent of one another, $\prod_{\ell} \mathrm{OPT}_{\ell}$ is an upper bound on maximum probability OPT, over any placement of $N$, that the number of failed tasks due to failures at all levels is at most $F$. Here, we have used the fact that for the total number of failures to be at most $F$, the number of failures at each level need to be at most $F$. We thus obtain

$$
\begin{aligned}
\operatorname{Pr}[X(S) \leq(L+\varepsilon) F / N] & \geq \prod_{\ell} P_{\ell}\left(X_{\ell}(S) \leq(1+\varepsilon / L) F / N\right) \\
& \geq \prod_{\ell}\left(\mathrm{OPT}_{\ell}-O(\varepsilon / L)\right) \\
& \geq \mathrm{OPT}-O(\varepsilon)
\end{aligned}
$$

where in the second step, we invoke Equation (3), with $\varepsilon$ replaced by $\varepsilon / L$. This gives us Theorem 1.10 , restated below.

Theorem 5.5 (Restatement of Theorem 1.10). For any $\varepsilon>0$, there exists a polynomial time algorithm for HIERPROBFAP over an L-level hierarchy that runs in time $n^{\text {poly }(L, 1 / \varepsilon)}$ and returns a feasible placement such that

$$
\operatorname{Pr}[\text { number of failed tasks } \leq(L+\varepsilon) F] \geq \mathrm{OPT}-O(\varepsilon),
$$

where OPT is the maximum probability, over any placement of $N$ tasks, that the number of failed tasks is at most $F$.

\section{CONCLUSIONS AND OPEN PROBLEMS}

In this article, we formulate and initiate the study of failure-aware placement for hierarchical data centers under adversarial and probabilistic failures. For both classes, we give hardness results and approximation algorithms for multiple variants: based on generalized spreading for HIERRoBUsTFAP and Poisson approximation for HierProBFAP.

One natural question is to improve the approximation guarantees and design more practical implementations for some of the variants. In particular, the approximation factor in the bicriteria PTAS for HiERPRoBFAP grows linearly in the number of levels. While this is reasonable for current datacenters (which typically have three to four levels of hierarchy), it would be interesting to see if the approximation factor can be made sublinear or even independent of number of levels. Another useful area is to design practical implementations of PTASes for RoBUSTFAP and bicriteria PTASes for ProbFAP and HierProbFAP to trade off approximation guarantees for simplicity.

Our analysis so far has focused on tree topologies and placing multiple tasks of a single job. An interesting open question is to extend this to other topologies and an online sequence of jobs possibly of different sizes. A further extension would be to consider dynamic task migrations to improve availability as tasks finish and leave. 


\section{ACKNOWLEDGMENTS}

We would like to thank Gerhard Woeginger for providing insights into the results of Caprara et al. (2013) and sharing a full version of their paper. We also thank the anonymous referees for helping improve the quality of this article.

\section{REFERENCES}

Ceph storage architecture. Retrieved January 17, 2018 from http://docs.ceph.com/docs/giant/architecture/.

S. Agrawal, Y. Ding, A. Saberi, and Y. Ye. 2012. Price of correlations in stochastic optimization. Operations Research 60 (February 2012), 243-248.

Mohammad Al-Fares, Alexander Loukissas, and Amin Vahdat. 2008. A scalable, commodity data center network architecture. ACM SIGCOMM Computer Communication Review 38, 63-74.

Luiz André Barroso and Urs Hölzle. 2009. The datacenter as a computer: An introduction to the design of warehouse-scale machines. Synthesis Lectures on Computer Architecture 4 (2009), 1-108.

D. Bertsimas and M. Sim. 2004. The price of robustness. Operations Research 52, 1 (2004), 35-53.

A. Bhalgat, A. Goel, and S. Khanna. 2011. Improved approximation results for stochastic knapsack problems. In SODA.

P. Bodik, I. Menache, M. Chowdhury, P. Mani, D. A. Maltz, and I. Stoica. 2012. Surviving failures in bandwidth-constrained datacenters. In Proceedings of the ACM SIGCOMM 2012 Conference on Applications, Technologies, Architectures, and Protocols for Computer Communication.

Alberto Caprara, Margarida Carvalho, Andrea Lodi, and Gerhard J. Woeginger. 2013. A complexity and approximability study of the bilevel knapsack problem. In Integer Programming and Combinatorial Optimization. 98-109.

Min Chen, Shiwen Mao, and Yunhao Liu. 2014. Big data: A survey. Mobile Networks and Applications 19, 2 (2014), 171-209.

C. Daskalakis, A. De, I. Diakonikolas, A. Moitra, and R. Servedio. 2014. A polynomial-time approximation scheme for fault-tolerant distributed storage. In SODA.

J. Dean and S. Ghemawat. 2008. MapReduce: Simplified data processing on large clusters. Communications of the ACM 51 (2008), 107-113.

Christina Delimitrou and Christos Kozyrakis. 2013. QoS-aware scheduling in heterogeneous datacenters with paragon. ACM Transactions on Computer Systems (TOCS) 4 (2013), 12:1-12:34.

A. Demers, S. Keshav, and S. Shenker. 1989. Analysis and simulation of a fair queueing algorithm. SIGCOMM Computer Communication Review 19, 4 (Aug. 1989), 1-12.

S. Dempe. 2002. Foundations of Bilevel Programming. Kluwer Academic Publishers.

S. Dempe and K. Richter. 2000. Bilevel programming with knapsack constraint. Central European fournal of Operations Research 8, 2 (2000), 93-107.

S. DeNegre. 2011. Interdiction and Discrete Bilevel Linear Programming. Ph.D. Dissertation. Lehigh University.

D. Ford, F. Labelle, F. I. Popovici, M. Stokely, V. A. Truong, L. Barroso, C. Grimes, and S. Quinlan. 2010. Availability in globally distributed storage systems. In Proceedings of Operating Systems Design and Implementation (OSDI).

M. R. Garey and D. S. Johnson. 1979. Computers and Intractability: A Guide to the Theory of NP-Completeness. Freeman, New York.

P. Gill, N. Jain, and N. Nagappan. 2011. Understanding network failures in data centers: Measurement, analysis, and implications. ACM SIGCOMM Computer Communication Review.

M. Gribaudo, M. Iacono, and D. Manini. 2015. Improving reliability and performances in large scale distributed applications with erasure codes and replication. Future Generation Computer Systems (2015).

S. Keshav. 1997. An Engineering Approach to Computer Networking: ATM Networks, the Internet, and the Telephone Network. Addison-Wesley Longman Publishing Co., Inc., Boston, MA.

O. Klopfenstein and D. Nace. 2008. A robust approach to the chance-constrained knapsack problem. Operations Research Letters 36, 5 (2008), 628-632.

Madhukar Korupolu and Rajmohan Rajaraman. 2016. Robust and probabilistic failure-aware placement. In Proceedings of the ACM Symposium on Parallelism in Algorithms and Architectures.

L. Le Cam. 1960. An approximation theorem for the Poisson binomial distribution. Pacific Journal of Mathematics 10 (1960), $1181-1197$.

Charles E. Leiserson. 1985. Fat-trees: Universal networks for hardware-efficient supercomputing. IEEE Transactions on Computing 10 (Oct. 1985), 892-901.

J. Li and A. Deshpande. 2011. Maximizing expected utility for stochastic combinatorial optimization problems. In FOCS.

J. Li and W. Yuan. 2013. Stochastic combinatorial optimization via Poisson approximation. In STOC.

Florence Jessie MacWilliams and Neil James Alexander Sloane. 1977. The Theory of Error-correcting Codes. Vol. 16. Elsevier.

R. Mansi, C. Alves, J. de Carvalho, and S. Hanafi. 2012. An exact algorithm for bilevel 0-1 knapsack problems. Mathematical Problems in Engineering (2012). 
K. A. Mills, R. Chandrasekaran, and N. Mittal. 2015. Algorithms for replica placement in high-availability storage. In ArXiv Preprint arXiv:1503.02654.

J. Nagle. 1987. On packet switches with infinite storage. IEEE Transactions on Communications 35, 4 (1987), 435-438.

A. Nemirovski and A. Shapiro. 2006. Convex approximations of chance constrained programs. SIAM fournal of Optimization 17, 4 (2006), 969-996.

E. Nikolova. 2010. Approximation algorithms for offline risk-averse combinatorial optimization. In APPROX.

B. K. Pagnoncelli, S. Ahmed, and A. Shapiro. 2009. Sample average approximation method for chance-constrained programming: Theory and applications. Journal of Optimization Theory and Applications 142, 2 (2009), 399-416.

A. Parekh and R. Gallager. 1993. A generalized processor sharing approach to flow control in integrated services networks: The single-node case. IEEE/ACM Transactions on Networking 1, 3 (1993), 344-357.

Eduardo Pinheiro, Wolf-Dietrich Weber, and Luiz André Barroso. 2007. Failure trends in a large disk drive population.. In FAST. 17-23.

James S Plank and others. 1997. A tutorial on reed-solomon coding for fault-tolerance in RAID-like systems. Software: Practice and Experience 27, 9 (1997), 995-1012.

C. Reiss, A. Tumanov, G. Ganger, R. Katz, and M. Kozych. 2012. Heterogeneity and dynamicity at scale: Google trace analysis. In Proceedings of the ACM Symposium on Cloud Computing.

Bianca Schroeder and Garth A Gibson. 2007. Understanding failures in petascale computers. fournal of Physics: Conference Series 78, 012022 .

B. Schroeder, E. Pinheiro, and W. D. Weber. 2009. Dram errors in the wild: A large-scale field study. ACM SIGMETRICS Performance Evaluation Review 37, 1 (2009), 193-204.

M. Sedaghat, E. Wadbro, J. Wilkes, S. De Luna, O. Seleznjev, and E. Elmroth. 2016. Die-hard: Reliable scheduling to survive correlated failures in cloud data centers. In Proceedings of IEEE/ACM International Symposium on Cluster, Cloud and Grid Computing (CCGrid'16).

D. Tang and R. K. Iyer. 1992. Analysis and modeling of correlated failures in multicomputer systems. IEEE Transactions on Computers 41, 5 (1992), 567-577.

A. Verma, L. Pedrosa, M. Korupolu, D. Oppenheimer, E. Tune, and J. Wilkes. 2015. Large-scale cluster management at Google with Borg. In Proceedings of the European Conference on Computer Systems (EuroSys'15).

K. V. Vishwanath and N. Nagappan. 2010. Characterizing cloud computing hardware reliability. In Proceedings of the ACM Symposium on Cloud Computing (SOCC'11).

H. von Stackelberg. 1952. The Theory of Market Economy. Oxford University Press.

Sage A. Weil, Scott A. Brandt, Ethan L. Miller, and Carlos Maltzahn. 2006. CRUSH: Controlled, scalable, decentralized placement of replicated data. In Proceedings of the 2006 ACM/IEEE Conference on Supercomputing (SC'06).

P. Yalagandula, S. Nath, H. Yu, P. B. Gibbons, and Seshan S.2004. Beyond availability: Towards a deeper understanding of machine failure characteristics in large distributed systems. In Proceedings of the Workshop on Real, Large Distributed Systems (WORLDS'04).

M. Zaharia, M. Chowdhury, M. J. Franklin, S. Shenker, and I. Stoica. 2010. Spark: Cluster computing with working sets. In Proceedings of the 2nd USENIX Conference on Hot Topics in Cloud Computing.

Received December 2016; revised January 2018; accepted March 2018 